\title{
Bouncing Quantum Cosmology
}

\author{
Nelson Pinto-Neto [D
}

check for

updates

Citation: Pinto-Neto, N. Bouncing Quantum Cosmology. Universe 2021, 7, 110. https://orcid.org/10.3390/ universe7040110

Academic Editors: Yi-Fu Cai,

Chunshan Lin and Antonino Marciano

Received: 14 March 2021

Accepted: 12 April 2021

Published: 20 April 2021

Publisher's Note: MDPI stays neutral with regard to jurisdictional claims in published maps and institutional affiliations.

Copyright: (c) 2021 by the authors. Licensee MDPI, Basel, Switzerland. This article is an open access article distributed under the terms and conditions of the Creative Commons Attribution (CC BY) license (https:// creativecommons.org/licenses/by/ $4.0 /)$.
Centro Brasileiro de Pesquisas Físicas, Rua Dr. Xavier Sigaud 150, Urca, Rio de Janeiro 22290-180, RJ, Brazil; nelsonpn@cbpf.br; Tel.: +55-21-21417381

\begin{abstract}
The goal of this contribution is to present the properties of a class of quantum bouncing models in which the quantum bounce originates from the Dirac canonical quantization of a midi-superspace model composed of a homogeneous and isotropic background, together with small inhomogeneous perturbations. The resulting Wheeler-DeWitt equation is interpreted in the framework of the de Broglie-Bohm quantum theory, enormously simplifying the calculations, conceptually and technically. It is shown that the resulting models are stable and they never get to close to the Planck energy, where another more involved quantization scheme would have to be evoked, and they are compatible with present observations. Some physical effects around the bounce are discussed, like baryogenesis and magnetogenesis, and the crucial role of dark matter and dark energy is also studied.
\end{abstract}

Keywords: bounce; quantum cosmology; inflation; cosmological perturbations; stability; dark energy

\section{Introduction}

Cosmological observations (the cosmic microwave background radiation [1], Big Bang nucleosynthesis [2], large scale structure [3] and cosmological red-shift [4]) strongly indicate that the Universe is expanding from a very hot era, when the geometry of space was highly homogeneous and isotropic (maximally symmetric space-like hyper-surfaces), with tiny deviations from this special symmetric state.

Cosmology is a very peculiar part of Physics, because the physical system under investigation is the Universe as a whole, and one cannot control its initial conditions, as in other physical situations. Hence, finding physical justifications for the Universe's initial conditions becomes an important part of its physical understanding, along with setting the dynamical equations that the degrees of freedom describing it must obey, which are generally assumed to be given by General Relativity (GR).

Without a consensus theory of quantum gravity, it is difficult to construct a measure on the configuration space of GR. However, generically, field theories contain $d$ degrees of freedom per space point $\left(d \times \infty^{3}\right.$ degrees of freedom), hence initial homogeneity requires that $\infty^{3}$ degrees of freedom have very close initial values, see References [5-7] for discussions about this issue. Consequently, as GR is a field theory, solutions of GR with maximally symmetric space-like hyper-surfaces are very special, even when the matter content distribution is homogeneous and isotropic. This is because the matter energymomentum tensor determines only the Ricci tensor, through Einstein's equations, while the Weyl tensor (the conformal curvature tensor) remains completely undetermined. For instance, a null energy-momentum tensor is trivially homogeneous and isotropic, and it is widely known that there are many inhomogeneous solutions of Einstein's equation in vacuum, the first and iconic one being the Schwarzschild solution. Hence, only through very special initial conditions for the dynamical degrees of freedom, which, of course, must also contain the spatial geometry and its time derivative (the extrinsic curvature), a space-time geometry with maximally symmetric space-like hyper-surfaces can emerge. Another possibility is that such special space-time solutions are attractors of the dynamical equations once some general physical conditions are assumed. Both attempts have not succeeded up to now. The first one relies essentially in constructing a theory of initial 
conditions, presumably coming from quantum gravity and quantum cosmology. However, there is no consensus on an accepted theory of quantum gravity and quantum cosmology, which are under construction by many independent groups [8-13]. The second one relies on numerical computations assuming some physical frameworks (like inflation [14], or ekpyrotic contraction [15]), but they run into difficulties due to the high complexity of Einstein's equations, forcing them, in the end, to strongly restrain the initial conditions anyway, although in a less severe manner. It seems to me that this second approach suffers from a serious conceptually flaw: given all fields and particles of the standard model of particle physics, GR allows infinitely many inhomogeneous cosmological solutions which, of course, cannot be framed back to the past highly hot and maximally symmetric space indicated by cosmological observations. I suppose that only anthropic arguments [16] can retrieve this alternative.

Besides initial condition issues, the standard cosmological model suffers from a major flaw-extrapolating GR back to the past, one necessarily encounters a singularity, where physical quantities, like the curvature of space-time, the matter energy density, among others, diverge. Hence, the model is incomplete. Attempts to eliminate the initial cosmological singularity rely on classical corrections of General Relativity, or on quantum effects. There are two possibilities: either this new physics connects the expanding Universe to a physically sensible preceding era through its very hot and dense phase, or there is some sort of non-singular beginning, like the quantum tunneling from nothing [17], or the no-boundary proposal [18]).

If one assumes that the Universe had a beginning immediately followed by the hot expanding phase discussed above, then some new important puzzles concerning initial conditions in cosmology arise. It is usually assumed that this hot expanding phase is dominated by radiation, and then by pressureless matter. In this case, GR asserts that the expansion must be decelerated, implying that the distance scale of regions with causal contact in the Universe, given by the Hubble radius, $R_{H} \equiv 1 / H \equiv a / \dot{a}$, where $a$ is the scale factor and the dot represents derivative with respect to cosmic time, grows faster than all distance scales in the Universe, which are proportional to the scale factor itself:

$$
\frac{\mathrm{dR}_{\mathrm{H}}}{\mathrm{da}}=1-\frac{\ddot{a} a}{\dot{a}^{3}}>1,
$$

if $\ddot{a}<0$ and $\dot{a}>0$. This means that most of the scales we are able to see today were out of the Hubble radius in the past. In particular, the cosmic microwave background radiation (CMBR) we see in the sky today, with almost the same temperature in all directions, would have contained hundreds of causally disconnected regions when it was formed, precluding any causal physical understanding of its observed temperature isotropy (this is the so called horizon problem). Furthermore, it is not possible to get any viable physical explanation for the power spectrum of primordial cosmological perturbations, which evolved to form the structures we observe today, as long as the large scales of inhomogeneities of cosmological interest we see today were already bigger than the Hubble radius in the near past. Finally, it is observed that the curvature of the space-like hyper-surfaces today is almost null, or, equivalently, that the matter energy density of the Universe today, $\rho_{0}$, is very near the critical energy density $\rho_{c}$, which is the total matter energy the Universe would have if their space-like hyper-surfaces were flat: $\Omega_{0} \equiv \rho_{0} / \rho_{c} \approx 1$. However, the ratio $\Omega(t) \equiv \rho(t) / \rho_{c}$ evolves as

$$
\frac{\mathrm{d}|\Omega(t)-1|}{\mathrm{d} t}=-2 \frac{\ddot{a}}{\dot{a}^{3}} \text {. }
$$

Once again, the ratio $\ddot{a} / \dot{a}^{3}$ appears, and if $\ddot{a}<0$ and $\dot{a}>0$, then $\Omega(t)$ was astonishingly near unity near the beginning of the Universe, implying an spectacular fine tuning of initial conditions. This is the so called flatness problem. Hence, there is no possible causal physical explanation for the peculiar properties of the universe we observe, apart some yet unknown theory of initial conditions, if it had a beginning succeeded by a decelerated expanding phase. 
In order to get rid of these new puzzles, and keeping the hypothesis that the Universe had a beginning followed by an expanding phase, one possible way out is to assume that there was a field, called inflation, which dominated the dynamics of the Universe expansion before the radiation era. When the inflaton field does not satisfy the strong energy condition $(\rho+3 p \geq 0, \rho+p \geq 0$, where $p$ is the pressure), which is a commonplace feature of field theories, the expansion is accelerated, $\ddot{a} / a>0$, and the problems arising from Equations (1) and (2) are overcome. During this phase, called inflation [19-21], it is possible that $a$ increases faster than $R_{H}$, see Equation (1), and, looking backwards in time, scales of cosmological interest were smaller than the Hubble radius in this era, allowing causal contact, and avoiding the horizon problem and the structure formation issue. In the case of the flatness problem, Equation (2) shows that, during inflation, $\Omega(t)$ approaches unit from whatever value it would have in the beginning, eliminating the fine tuning. In intuitive terms, the picture is of a spherical surface that expands so much, that the part of it we can see is a tiny piece of the spherical surface, making it almost flat. Also, concerning the horizon problem, this inflationary expansion turns a very small part of the early Universe which had causal contact in the far past as big as the Universe we see today. Concluding, under these assumptions (the Universe had a beginning followed by an expanding phase), inflation is a necessary ingredient in order to yield the possibility of a causal physical explanation of some features of our Universe. Indeed, it gave a sound description of the origin of cosmological perturbations, the properties of their power spectra, and a confirmed prediction for the spectral index of scalar cosmological perturbations at large scales, $n_{s} \approx 0.97[22,23]$. It also leads to the isotropization of homogeneous hyper-surfaces (the so called Bianchi models, under certain special conditions in the case of positive curvature space-like hyper-surfaces). Concerning the homogeneity issue, however, it is important to emphasize that inflation indeed turns viable a dynamical explanation of it, but it does not yield the explanation itself, at least up to now, for the reasons I pointed out above.

Looking at Equations (1) and (2), instead of assuming that $\ddot{a}>0$ ( keeping $\ddot{a}<0$ ), one could get the same consequences supposing that $\dot{a}<0$, implying a contracting decelerating phase. The flatness and horizon problems are naturally solved in the same way. The intuitive picture is of a spherical surface which is shrinking, but the glasses we use to see the surface (the Hubble radius) yield images which are shrinking much faster, leading to the impression that the surface is becoming flat. Running backwards in time in this contracting decelerating phase, when the Universe was immensely large in the past, turn all cosmological scales of physical interest much smaller than the Hubble radius, solving the horizon and structure formation issues as well. It is a different mechanism that leads to the same results. However, a model with a contracting phase must be connected to the expanding phase we observe, performing a bounce. The occurrence of a bounce needs more stringent conditions to happen than inflation. Within GR, a realistic bounce can only happen if the field violates the null energy condition $(\rho+p \geq 0)$, which may be plagued by quantum instabilities. Hence, most of the bouncing models are obtained outside GR [24-33], or in the context of quantum cosmology [34-39], see also References [40,41] for some reviews. An important remark is that, once one finds a physical mechanism yielding a bounce, the overall cosmological model is generally not singular. Hence, the singularity problem is automatically solved.

As we have seen above, in bouncing models the horizon, flatness, and structure formation issues do not arise. Hence, they do not need an inflationary era, although they are not incompatible with it. In fact, if the investigation and construction of sensible bouncing models were realized long ago, putting aside the picture of a universe with a beginning followed by expansion, I doubt whether the idea of inflation would even be formulated. However, the history of cosmology is not like this. Bouncing models were indeed investigated in the past [42], but the theoretical difficulties to realize them at that time (unlimited entropy growth, new physics to realize the bounce, instabilities) induced physicists to think that it was simpler to think that the Universe had a beginning, postponing the discussion on how this beginning 
was realized. In this context, as we have seen above, inflation is very important, and an almost logical consequence of this assumption. Furthermore, inflation made predictions which were confirmed afterwards by observations in the CMBR (the spectral index, the acoustic peaks, small non-gaussianities [23]), supporting this hypothesis. Because of that, many cosmologists put inflation as a part of the standard cosmological model, and it has been investigated in many details since it was proposed. Meanwhile, investigations concerning bouncing models have been deepened progressively. The objections against them were refuted, many physical realizations of a bounce were formulated, and it has been shown that this models could also describe the features of our Universe, specially the ones associated with the CMBR. Note, however, that bouncing models have been able to be compatible with observations already made but, up to now, they did not make predictions which have been observed, like inflation.

The aim of this paper is to describe features and properties of bouncing models in more detail, and whether they can really be an alternative to inflation, or at least a complementation of it, as it solves, by construction, the singularity problem. I will focus in bouncing models where the singularity is solved through quantum effects coming from quantum cosmology. Other approaches will be discussed elsewhere in this volume. I will present some possible backgrounds, study the evolution of perturbations in such backgrounds, and other physical aspects of it, like baryogenesis. In Section 2, I will describe in some detail such backgrounds and the physical context in which they are obtained. In Section 3, I will present the evolution of cosmological perturbations in such backgrounds, and I will investigate the consequences of the contracting phase and the bounce to the features of the CMBR. I will focus on the so called matter bounce, which leads to an almost scale invariant power spectrum of scalar perturbations. I will also discuss primordial gravitational waves in this context. In Section 4, I will show that these models are stable under scalar, vector, and tensor perturbations. In Section 5, I will discuss the role of dark energy in bouncing models, a very important aspect of these models which has not been investigated with the the care it deserves. In Section 6, I will discuss, particle creation, magnetogenesis and bariogenesis in bouncing models. I will end up in Section 7, with conclusions and discussions.

\section{Bouncing Models from Quantum Cosmology}

The Copenhagen interpretation of quantum mechanics [43-45], which is the one taught in undergraduate courses and used by the majority of physicists in all areas, does not fit with a quantum theory of cosmology. This is because it imposes the existence of a classical domain beyond the quantum one, or of an external agent outside the physical system, by assuming that there is a fundamental process in a measurement which brings quantum potentialities to experimental facts through the collapse of the wave function. This process does not satisfy the unitary quantum evolution, and it must occur outside the quantum world, by an external agent in a classical domain. Of course, if we want to quantize the whole Universe, there is no place for a classical domain or any external agent outside it, and the Copenhagen interpretation cannot be applied.

This conceptual problem is solved by the de Broglie-Bohm (dBB) quantum theory (there are other alternatives, as the spontaneous collapse approach [46,47], the ManyWorlds Interpretation (MW) [48], which will not be discussed here). In dBB theory, a system is not only described by the wave function, but also by extra variables [49-51]. In the context of non-relativistic $\mathrm{dBB}$ mechanics, there are actual particles whose motion is determined by the wave function, and these extra variables are their particle positions. Usually, in a measurement process, the total wave function splits into many branches, each one associated with one possible result of measurement. The point particle in configuration space describing the whole system will enter into one of the branches (depending on the initial position of the point particle before the measurement, which is unknown), and the other branches will be empty. It can be shown [50] that the empty waves can neither interact with other particles, nor with the point particle containing the apparatus. Hence, no observer can be aware of the other branches which are empty. One gets an effective 
but not real collapse (the empty waves continue to exist), and quantum unitary evolution is universally valid. The probabilistic character of Quantum Mechanics is due to the ignorance about the initial position of the point particle, arising statistically, not being intrinsic to the theory. The dBB quantum theory can also be extended to quantum field theory [52], where the extra variables may be particles or fields, and to canonical quantum gravity [53-55]. In the context of quantum gravity, the extra variables are the space metric, and whatever suitable variable for the matter fields. Therefore, an external observer is no longer needed, and we achieve the conceptual coherence necessary to apply this approach to cosmology.

Canonical quantum gravity is the most conservative approach to quantum gravity. It is obtained by applying the usual quantization techniques, which were so successful in high energy physics, to GR. The quantization starts with passing from the Lagrangian to the Hamiltonian picture, and then mapping Poisson brackets to commutation relations of operators. As GR is invariant under time and space reparametrizations, its Hamiltonian is a combination of constraints which, according to the Dirac quantization procedure, should annihilate the quantum state. Unfortunately, the final equation arising from this procedure, the Wheeler-DeWitt equation [56], is just a formal equation plagued with many mathematical problems. One alternative is to move to loop quantum gravity [10]. However, as the hot and dense universe was very homogeneous and isotropic in the past, as we have seen, it may be possible to restrict the allowed spatial geometries in the quantization scheme to be homogeneous with small inhomogeneous perturbations around it. Under this restriction, one can perform calculations with simplified equations, either within the framework of canonical quantum gravity, or in loop quantum gravity. This limited framework is called quantum cosmology $[13,38,39]$, and loop quantum cosmology [12], respectively. The complete connection of these simplifications with the full theories they come from is yet unknown. Nevertheless, one expects that at energy scales which are not so close to the Planck energy, these approaches can be applied as good approximations to the hot and dense universe, yielding sensible results. We will restrict ourselves here to quantum cosmology itself, viewed in the framework of the dBB quantum theory, the loop quantum cosmology approach being treated elsewhere.

The non-relativistic $\mathrm{dBB}$ theory is about point-particles in physical space moving under the influence of the wave function [49-51]. The equations of motion for the configuration $X=\left(\mathbf{X}_{1}, \ldots, \mathbf{X}_{n}\right)$ of the particles, called the guidance equation, is given by (Throughout the paper we assume units in which $\hbar=c=1$.)

$$
\dot{X}(t)=v^{\psi}(X(t), t),
$$

where $v^{\psi}=\left(\mathbf{v}_{1}^{\psi}, \ldots, \mathbf{v}_{n}^{\psi}\right)$, with

$$
\mathbf{v}_{k}^{\psi}=\frac{1}{m_{k}} \operatorname{Im}\left(\frac{\nabla_{k} \psi}{\psi}\right)=\frac{1}{m_{k}} \nabla_{k} S
$$

and $\psi=|\psi| e^{i S}$. Solutions of Equation (3) are called Bohmian trajectories. The wave function $\psi(x, t)=\psi\left(\mathbf{x}_{1}, \ldots, \mathbf{x}_{n}\right)$ itself satisfies the non-relativistic Schrödinger equation

$$
i \partial_{t} \psi(x, t)=\left(-\sum_{k=1}^{n} \frac{1}{2 m_{k}} \nabla_{k}^{2}+V(x)\right) \psi(x, t) .
$$

For an ensemble of systems with the same wave function $\psi$, a particle distribution given by $\left|\psi\left(x, t_{0}\right)\right|^{2}$ at some time $t_{0}$ will be given by $|\psi(x, t)|^{2}$ at all times $t$. This is because any distribution $\rho$ transported by the particle motion satisfies the continuity equation

$$
\partial_{t} \rho+\sum_{k=1}^{n} \nabla_{k} \cdot\left(\mathbf{v}_{k}^{\psi} \rho\right)=0
$$


while $|\psi|^{2}$ satisfies the same equation, that is,

$$
\partial_{t}|\psi|^{2}+\sum_{k=1}^{n} \nabla_{k} \cdot\left(\mathbf{v}_{k}^{\psi}|\psi|^{2}\right)=0,
$$

which is a consequence of the Schrödinger equation.

These are called equilibrium distributions [51]. Non-equilibrium distributions would lead to a deviation of the Born rule. However, they generally tends to equilibrium distributions in a coarse grained level, see References $[57,58]$. Therefore, except for these interesting possibilities, the $\mathrm{dBB}$ theory reproduces the standard quantum predictions.

The dBB mechanics allows for an unambiguous characterization of the classical limit. Taking the time derivative of (3), we find that

$$
m_{k} \ddot{\mathbf{X}}_{k}(t)=-\left.\nabla_{k}\left(V(x)+Q^{\psi}(x, t)\right)\right|_{x=X(t)},
$$

where

$$
Q^{\psi}=-\sum_{k=1}^{n} \frac{1}{2 m_{k}} \frac{\nabla_{k}^{2}|\psi|}{|\psi|}
$$

is the quantum potential. Hence, if the quantum force $-\nabla_{k} Q^{\psi}$ is negligible compared to the classical force $-\nabla_{k} V$, then the $k$-th particle approximately moves along a classical trajectory.

Another aspect of the theory is that it allows for a simple and natural definition for the wave function of a subsystem [59]. Consider a system with wave function $\psi(x, y)$ where $x$ is the configuration variable of the subsystem and $y$ is the configuration variable of its environment. The actual configuration is $(X, Y)$, where $X$ is the configuration of the subsystem and $Y$ is the configuration of the other particles. The wave function of the subsystem $\chi(x, t)$, called the conditional wave function, is then defined as

$$
\chi(x, t)=\psi(x, Y(t), t) .
$$

This is a natural definition since the trajectory $X(t)$ of the subsystem satisfies

$$
\dot{X}(t)=v^{\psi}(X(t), Y(t), t)=v^{\chi}(X(t), t) .
$$

Hence, for the evolution of the subsystem's configuration, we can either use the conditional wave function or the total wave function (keeping the initial positions fixed). The time evolution of the conditional wave function does not necessarily satisfy a Schrödinger equation, although in many cases it does. The conditional wave function collapses during measurement situations, which constitutes an explanation for the success of the collapse postulate in standard quantum mechanics. In the context of quantum cosmology, the conditional wave function will be used to derive an effective Schrödinger equation for a subsystem of the universe, the one associated with inhomogeneous cosmological perturbations around a homogeneous background, which is derived from a time-independent universal wave function.

The dBB quantum theory can be extended to quantum field theory. In this case, field configurations are usually attributed with objective reality, and their time evolutions are given by guidance equations in the same way as in non-relativistic quantum mechanics. The probabilistic predictions are identical as in quantum field theory, hence they are Lorentz invariant. However, the Bohmian evolution of the fields is not generally Lorentz invariant, their inner symmetries depend on the particular wave functional solution, and they can be calculated, see Reference [55]. Nevertheless, such Bohmian evolutions are hidden from experimental scrutiny in any quantum equilibrium situation, as discussed above.

Note that there are other approaches to investigate quantum gravity effects, where part of the whole system can be treated classically, either by integrating out the quantum variables to calculate their mean effects in the classical variables, see Reference [60], or 
to use the classical background degrees of freedom to perform a Born-Oppenheimer approximation, and transform the Wheeler-DeWitt equation into a Schrödinger equation for the quantum variables [61]. Both of them rely on the existence of "classical" variables, hence they cannot treat the case where all degrees of freedom are quantum. Also, even in the presence of classical variables, when the system is the Universe, one will have to ultimately face the problem of the interpretation of quantum theory, or how a single reality emerges from the different possibilities allowed by the wave functional without a collapse postulate. One good example of this acute problem is the quantum-to-classical transition of quantum cosmological perturbations in classical backgrounds, which demands a much deeper understanding that is not contemplated by these approaches, see References [62-65]. In the de Broglie-Bohm quantum theory, the assumption of objective reality of the Bohmian trajectories allow to perform calculations even when all degrees of freedom are quantum, and yields an explanation for the emergence of a single classical reality, as explained above [51], and in References [66,67] for the case of the quantum-to-classical transition of quantum cosmological perturbations in classical backgrounds. Of course, it is expected that when the Bohmian trajectories for some degrees of freedom become classical, the two approaches above can be applied and coincide $[66,67]$.

One of the important results of the application of the dBB quantum theory to quantum cosmology is the elimination of cosmological singularities, which generically appears in classical GR [68], as we will now see.

\subsection{The Canonical Scalar Field}

In this subsection, I will assume that the matter content of the Universe can be described by a canonical scalar field $\varphi$. As discussed above, from all possible space geometries and scalar field configurations (called superspace), we will restrict ourselves to homogeneous and isotropic configurations (called mini-superspace). The inhomogeneous perturbations will be treated in the next sections. Hence, the model is given by the homogeneous and isotropic Friedmann-Lemaître-Robertson-Walker (FLRW) metric coupled to a homogeneous scalar field. The metric reads

$$
\mathrm{d} s^{2}=N(t)^{2} \mathrm{~d} t^{2}-a(t)^{2} \mathrm{~d} \Omega_{k}^{2}
$$

where $N$ is the lapse function, $a$ is the scale factor, and $\mathrm{d} \Omega_{k}^{2}$ is the spatial line-element on three-space with constant curvature. We are using natural units $\hbar=c=1$.

The GR equations are obtained from the Einstein-Hilbert Lagrangian which, for geometries of type (12) and a homogeneous scalar field $\varphi$, reduces to

$$
L=N a^{3} \kappa\left[-\frac{\dot{a}^{2}}{2 a^{2} N^{2}}-V_{G}+\kappa^{2}\left(\frac{\dot{\varphi}^{2}}{2 N^{2}}-V_{M}\right)\right],
$$

where $\kappa=\sqrt{4 \pi G / 3} \equiv l_{\mathrm{P}} / \sqrt{6}$, with $G$ being the gravitational constant, $l_{\mathrm{P}}$ is our definition of the Planck length, $V_{M}$ is the potential for the scalar field, $V_{G}=-k /\left(2 l^{2} a^{2}\right)+\Lambda / 6$, $k=0, \pm 1$ for flat, positively or negatively curved space-like hyper-surfaces with constant curvature scale $l$, respectively, and $\Lambda$ is the cosmological constant. Note that we have redefined the scale factor as $\tilde{a}=a V^{1 / 3} / \kappa$. The quantity $V$ is the volume of the co-moving space-like hyper-surface, which appears due to the integration of the original EinsteinHilbert action in space. (As the co-moving space-like hyper-surface is homogeneous, its volume is a fixed constant, which was absorbed in the redefinition of $a$. In the case of compact hyper-surfaces, they are finite constants. For non-compact hyper-surfaces, one can integrate in a fixed box). Note that Lagrangian (13) has physical dimensions of length ${ }^{-1}$, compatible with our choice of natural units (remember that the scalar field $\varphi$ also has physical dimension length ${ }^{-1}$ ). 
Moving to the variable $\alpha=\ln a$, and performing Legendre transformations, one gets the Hamiltonian

$$
H \equiv N \mathcal{H}=\frac{N}{\mathrm{e}^{3 \alpha} \mathcal{\kappa}}\left[-\frac{\Pi_{\alpha}^{2}}{2}+\frac{\Pi_{\varphi}^{2}}{2 \kappa^{2}}+\mathrm{e}^{6 \alpha} \kappa^{2}\left(V_{G}+\kappa^{2} V_{M}\right)\right],
$$

where $\Pi_{\alpha}$ and $\Pi_{\varphi}$ are the momenta canonically conjugate to $\alpha$ and $\varphi$, respectively, and $N$ plays the role of a Lagrangian multiplier. Note that in the full Hamiltonian formalism of GR one gets the Hamiltonian and super-momentum density first class constraints, arising due to its invariance under time and space reparametrizations [56]. When one is restricted to homogeneous spatial hypersurfaces, the super-momentum density constraint is identically null, and the Hamiltonian density contains only the Hamiltonian constraint $\mathcal{H}$.

From the canonical relations

$$
\dot{\varphi}=\frac{N}{\kappa^{3} e^{3 \alpha}} \Pi_{\varphi}, \quad \dot{\alpha}=-\frac{N}{\kappa e^{3 \alpha}} \Pi_{\alpha}
$$

one gets the classical equations of motion

$$
\begin{gathered}
\frac{d}{d t}\left(\frac{\mathrm{e}^{3 \alpha} \dot{\varphi}}{N}\right)+N \mathrm{e}^{3 \alpha} \partial_{\varphi} V_{M}=0, \\
\frac{\dot{\alpha}^{2}}{N^{2}}=2 \kappa^{2}\left(\frac{\dot{\varphi}^{2}}{2 N^{2}}+V_{M}\right)+2 V_{G} .
\end{gathered}
$$

The first equation is the usual dynamical equation for a scalar field in a Friedmann geometry, and the second one is the Friedmann equation. The acceleration equation, which corresponds to the second-order equation for $\alpha$, follows from (16) and (17).

The Dirac quantization procedure imposes that the operator version of $\mathcal{H}$ must annihilate the quantum state $\psi, \hat{\mathcal{H}} \psi=0$, leading to the Wheeler-DeWitt equation

$$
\left[-\frac{\partial_{\alpha}^{2}}{2}+\frac{\partial_{\varphi}^{2}}{2 \kappa^{2}}-\mathrm{e}^{6 \alpha} \kappa^{2}\left(V_{G}+\kappa^{2} V_{M}\right)\right] \psi(\varphi, \alpha)=0,
$$

where, as usual, $\hat{\Pi}_{\alpha}=-i \partial_{\alpha}$, and $\hat{\Pi}_{\varphi}=-i \partial_{\varphi}$.

In the dBB theory, there is an actual scalar field $\varphi$, and an actual FLRW metric of the form (12), whose time evolutions are determined by

$$
\dot{\varphi}=\frac{N}{\kappa^{3} e^{3 \alpha}} \partial_{\varphi} S, \quad \dot{\alpha}=-\frac{N}{\kappa e^{3 \alpha}} \partial_{\alpha} S .
$$

It follows from these equations that

$$
\begin{gathered}
\frac{d}{d t}\left(\frac{\mathrm{e}^{3 \alpha} \dot{\varphi}}{N}\right)+N \mathrm{e}^{3 \alpha} \partial_{\varphi}\left(V_{M}+Q_{M}+Q_{G}\right)=0, \\
\frac{\dot{\alpha}^{2}}{N^{2}}=2 \kappa^{2}\left(\frac{\dot{\varphi}^{2}}{2 N^{2}}+V_{M}+Q_{M}\right)+2\left(V_{G}+\kappa^{2} Q_{G}\right),
\end{gathered}
$$

where

$$
Q_{M}=-\frac{1}{2 \kappa^{6} \mathrm{e}^{6 \alpha}} \frac{\partial_{\varphi}^{2}|\psi|}{|\psi|}, \quad Q_{G}=\frac{1}{2 \kappa^{4} \mathrm{e}^{6 \alpha}} \frac{\partial_{\alpha}^{2}|\psi|}{|\psi|}
$$

are, respectively, the matter and the gravitational quantum potentials. These equations differ from the classical ones by the quantum potentials.

In order to simplify the discussion about the singularities, let us consider the case of a free massless scalar field in flat space geometries $(k=0)$, without a cosmological constant. (Note that such a choice has a physical motivation. It can be a suitable model for the matter content in a very hot and dense universe, as it represents stiff matter, which is so 
dense that the speed of sound and light become equal: $p=\rho$ ). In this case, $V_{M}=V_{G}=0$. Working with the new dimensionless scalar field $\tilde{\varphi}=\kappa \varphi$, and omitting the tilde, the classical equations read

$$
\dot{\varphi}=\frac{N}{\kappa \mathrm{e}^{3 \alpha}} c, \quad \dot{\alpha}= \pm \frac{N}{\kappa \mathrm{e}^{3 \alpha}} c,
$$

where $c$ is an integration constant. Considering $c \neq 0$ (the case $c=0$ is the trivial flat Minkowski space-time), we have

$$
\alpha= \pm \varphi+\bar{c},
$$

with $\bar{c}$ another integration constant. In the plane $(\alpha, \varphi)$, these are straight lines with angular coefficient \pm 1 representing either universes expending indefinitely from the singularity $\alpha \rightarrow-\infty(a=0)$, or contracting from infinity to a singularity.

In the standard quantum mechanical approach to the Wheeler-DeWitt equation, the complete description is given by the wave function and, as mentioned in the Introduction, the notion of a singularity becomes ambiguous. This is not the case in the dBB theory, which describes the evolution of actual metric and matter fields. In this framework, the characterization of singularities is as simple and direct as in the classical case-they appear whenever the curvature of space-time, and/or the energy-momentum of the matter fields diverge. In the homogeneous and isotropic case, it happens when $a=0$.

The question of singularities in the free scalar field case was considered in Ref. [69]. In this case, the Wheeler-DeWitt equation reads

$$
\partial_{\varphi}^{2} \psi-\partial_{\alpha}^{2} \psi=0 .
$$

The solutions are

$$
\psi=\psi_{R}(\alpha-\varphi)+\psi_{L}(\alpha+\varphi) \equiv \psi_{R}(u)+\psi_{L}(v),
$$

where we have defined the light-cone coordinates $u, v$.

The actual metric may be non-singular; it depends on the wave function and on the initial conditions. For example, for a real wave function, $S=0$, the universe is static, so that there is no singularity. On the other hand, for complex wave functions $\psi=\psi_{R, L}$ the solutions are always classical. This is because one necessarily gets a total phase of the form $S=S(\alpha \pm \varphi)$, which are the general solutions of the classical Hamilton-Jacobi equation of the model, yielding the usual classical trajectories. Interesting wave function solutions are those leading to non-singular dynamical solutions which approach the classical limit for $\alpha>>1$ : universes with classical contracting and expanding phases, connected by a regular bounce. Note that any wave function with $\psi_{R}=-\psi_{L} \equiv F$ satisfies $\psi(\varphi, \alpha)=-\psi(\varphi,-\alpha)$, hence $\psi(\varphi, \alpha=0)=0$. As no Bohmian trajectory can cross a nodal point of the wave function, then there is no trajectory crossing the plane $\alpha=0$ in $(\alpha, \varphi)$-space. Hence, trajectories starting with $\alpha(0)>0$ will not have singularities. In this way, bouncing solutions can be obtained. Furthermore, if $F$ is square integrable, then, for $\alpha>>1$, either $F(u)$ or $F(v)$ will dominate in $\psi=F(u)-F(v)(F(u)$ for $\varphi>>1$, and $F(v)$ for $\varphi<<-1)$, leading to classical trajectories in these regions. Hence, in this case, the Bohmian trajectories represent universes contracting classically from the infinity past, experiencing a quantum bounce when it reaches its minimum volume, and then launched to a classical expanding phase. In Reference [69], a detailed picture of the Bohmian trajectories is displayed for the case that $F$ is a Gaussian.

In summary, this kind of model yields plenty of interesting bounce solutions, although singular models are also allowed, depending on the wave function and the initial conditions for the actual fields.

\subsection{Perfect Fluids}

Another example of a mini-superspace model is that of a FLRW space-time with a perfect fluid, where the pressure and the energy density satisfy the state equation $p=w \rho$, with $w$ constant. This kind of fluid may well describe the Universe at high temperatures, 
because fields and particles become highly relativistic, satisfying a radiation equation of state $p \approx \rho / 3$. I will now show that the Bohmian trajectories arising from the canonical quantization of this mini-superspace model also describe non-singular bouncing models.

A perfect fluid can be modelled by a scalar field as follows. Consider the matter Lagrangian

$$
L_{M}=\sqrt{-g} X^{n},
$$

where (In this section it is used dimensionless space-time coordinates $x^{\mu} \rightarrow x^{\mu} / \kappa$, a dimensionless scalar field $\varphi \rightarrow \kappa \varphi$, and a harmless redefinition of the scale factor, $a \rightarrow 2^{1 / 3} a$. Hence, the Lagrangian (27) is dimensionless because a dimensionless time parameter is now being used).

$$
X=\frac{1}{2} g^{\mu v} \partial_{\mu} \varphi \partial_{\nu} \varphi .
$$

I will assume that $X \geq 0$, and I will interpret $\varphi$ as the potential yielding the normalized $4-$ velocity of the fluid

$$
V_{\mu}=\frac{\partial_{\mu} \varphi}{(2 X)^{1 / 2}} .
$$

The energy-momentum tensor is given by

$$
T_{\mu \nu}=\frac{2}{\sqrt{-g}} \frac{\partial L_{M}}{\partial g^{\mu \nu}}=2 n X^{n} V_{\mu} V_{v}-g_{\mu v} X^{n} .
$$

Comparing with the usual expansion of the energy-momentum tensor in terms of energy density and pressure,

$$
T_{\mu v}=(\rho+p) V_{\mu} V_{v}-p g_{\mu v},
$$

We get

$$
p=X^{n}, \quad p=\frac{1}{2 n-1} \rho,
$$

implying that $w=1 /(2 n-1)$.

Assuming homogeneity, the scalar field depends only on time. The construction of the Hamiltonian is straightforward. The matter part reads

$$
H_{M}=c N \frac{p_{\varphi}^{1+w}}{a^{3 w}},
$$

where $p_{\varphi}$ is the momentum conjugate to $\varphi$ and $c=1 /\left(w \sqrt{2}^{1+3 w} n^{1+w}\right)$ is a constant.

Before implementing the canonical quantization procedure, I will perform the following canonical transformation:

$$
T=\frac{1}{c(1+w)} \frac{\varphi}{p_{\varphi}^{w}}, \quad P_{T}=c p_{\varphi}^{1+w},
$$

so that

$$
H_{M}=N \frac{P_{T}}{a^{3 w}} .
$$

An important property is that the momentum now appears linearly. Combining this perfect fluid Hamiltonian with the gravitational Hamiltonian for a FLRW geometry with flat space-like hyper-surfaces, the total mini-superspace Hamiltonian is obtained:

$$
H=N\left(-\frac{P_{a}^{2}}{4 a}+\frac{P_{T}}{a^{3 w}}\right) .
$$


It implies that $\dot{T}=N / a^{3 w}$ or, in terms of cosmic proper time $\tau, d T / d \tau=1 / a^{3 w}$. Hence, $T$ increases monotonically, so that it can be used as a clock variable. In terms of $T$, the scale factor evolves like $a \propto T^{2 / 3(1-w)}$, which is singular at $T=0$.

In the quantum case, because one momentum appears linearly in the Hamiltonian, the Wheeler-DeWitt equation assumes the Schrödinger form [38]

$$
\mathrm{i} \frac{\partial}{\partial T} \Psi(a, T)=\frac{1}{4}\left\{a^{(3 \omega-1) / 2} \frac{\partial}{\partial a}\left[a^{(3 \omega-1) / 2} \frac{\partial}{\partial a}\right]\right\} \Psi(a, T) .
$$

We adopted a factor ordering which makes Equation (37) covariant under redefinitions of $a$. The guidance equations are

$$
\dot{T}=\frac{N}{a^{3 w}}, \quad \dot{a}=-\frac{N}{2 a} \frac{\partial S}{\partial a} .
$$

The dynamics can be simplified using the transformation

$$
\chi=\frac{2}{3}(1-\omega)^{-1} a^{3(1-\omega) / 2},
$$

to obtain

$$
\mathrm{i} \frac{\partial \Psi(a, T)}{\partial T}=\frac{1}{4} \frac{\partial^{2} \Psi(a, T)}{\partial \chi^{2}} .
$$

This is just the time reversed Schrödinger equation for a one-dimensional free particle with mass 2 constrained to the positive axis.

In the context of standard quantum theory, the form of the Wheeler-DeWitt equation suggests to interpret $T$ as time and to find a suitable Hilbert space. Since $\chi>0$, the Hilbert space requires a boundary condition

$$
\left.\Psi\right|_{\chi=0}=\left.c \frac{\partial \Psi}{\partial \chi}\right|_{\chi=0},
$$

with $c$ a real constant. $\left|\Psi^{2}\right| d \chi$ is then the probability measure for the scale factor. The boundary condition makes that the total probability is preserved in time. In the dBB approach, the condition (41) implies that there are no singularities [70], because the condition means that the probability flux $J_{\chi} \sim \operatorname{Im}\left(\Psi^{*} \frac{\partial \Psi}{\partial \chi}\right)$ is null at $\chi=0$, so no trajectories can cross $a=0$ ). For wave functions not satisfying the boundary condition (41), however, singularities may be obtained, at least for some trajectories. For example, for a plane wave, the trajectories are the classical ones, hence a singularity is always obtained. From the dBB point of view this can motivate the consideration of a Hilbert space based on (41). It is then also natural to use $\left|\Psi^{2}\right| d \chi$ as the normalizable equilibrium distribution for the scale factor.

The dynamics of the scale factor can be expressed in terms of $T$ by solving the guiding Equation (38), which read,

or

$$
\frac{d a}{d T}=-\frac{a^{3 w-1}}{2} \frac{\partial S}{\partial a}
$$

$$
\frac{d \chi}{d T}=-\frac{1}{2} \frac{\partial S}{\partial \chi} .
$$

As an example of a wave function that satisfies condition (41), consider the initial Gaussian

$$
\Psi^{(\text {init })}(\chi)=\left(\frac{8}{T_{b} \pi}\right)^{1 / 4} \exp \left(-\frac{\chi^{2}}{T_{b}}\right),
$$


where $T_{b}$ is the constant variance of the Gaussian. The wave solution for all times in terms of $a$ is [? ]:

$$
\begin{aligned}
\Psi(a, T)=\left[\frac{8 T_{b}}{\pi\left(T^{2}+T_{b}^{2}\right)}\right]^{1 / 4} & \exp \left[\frac{-4 T_{b} a^{3(1-\omega)}}{9\left(T^{2}+T_{b}^{2}\right)(1-\omega)^{2}}\right] \\
& \times \exp \left\{-\mathrm{i}\left[\frac{4 T a^{3(1-\omega)}}{9\left(T^{2}+T_{b}^{2}\right)(1-\omega)^{2}}+\frac{1}{2} \arctan \left(\frac{T_{b}}{T}\right)-\frac{\pi}{4}\right]\right\} .
\end{aligned}
$$

The corresponding Bohmian trajectories are

$$
a(T)=a_{b}\left[1+\left(\frac{T}{T_{b}}\right)^{2}\right]^{\frac{1}{3(1-\omega)}} .
$$

Note that this solution has no singularities for whatever initial value of $a_{b} \neq 0$, and tends to the classical solution when $T \rightarrow \pm \infty$. The constant $T_{b}$ gives the time scale of the bounce. The solution (45) can be obtained for other initial wave functions [39].

For $w=1 / 3$ (radiation fluid), and adjusting the free parameters, the solution (45) can reach the classical evolution before the nucleosynthesis era, where the standard cosmological model starts to be compared with observations. Hence, it can be a good candidate to describe a sensible cosmological model at the radiation dominated era, which is free of singularities.

One can also include in this model a pressureless component, describing dark matter and baryons. This was done in Reference [70], yielding

$$
a\left(\eta_{s}\right)=a_{0}\left(\frac{\Omega_{m 0}}{4} \eta_{s}^{2}+\sqrt{\frac{1}{x_{b}^{2}}+\Omega_{r 0} \eta_{s}^{2}}\right) .
$$

In this expression, $x_{b}=a_{0} / a_{b}$ is the ratio between the scale factor today and the scale at the bounce, $a_{0}$ and $a_{b}$, respectively, $\Omega_{m 0}$ and $\Omega_{r 0}$ are the usual dimensionless densities $\left(\Omega=\rho / \rho_{c}\right)$ of dust and radiation, respectively. I have also defined the dimensionless conformal time, $\eta_{s}=\left(a_{0} / R_{H_{0}}\right) \eta$, where $R_{H_{0}}=1 / H_{0}$ is the Hubble radius today. The scale factor in Equation (46) describes a universe dominated by dust in the far past. As the universe contracts, radiation eventually dominates over dust, and near the bounce quantum effects become relevant. The quantum bounce happens, and it is followed by another radiation and dust phases. As we will see, the presence of dust is important not only for completeness, but also because it is necessary to yield a scale invariant spectrum of scalar perturbations.

Note that the Wheeler-DeWitt quantization we are using must be understood as an approximation of some complete theory of quantum gravity at scales not so close to the Planck length. The curvature scale at the bounce reads

$$
\left.L_{b} \equiv \frac{1}{\sqrt{R}}\right|_{\eta_{s}=0}=\left.\sqrt{\frac{a^{3}}{6 a^{\prime \prime}}}\right|_{\eta_{s}=0},
$$

where $R$ is the Ricci scalar. Using the values $H_{0}=70 \mathrm{~km} \mathrm{~s}^{-1} \mathrm{Mpc}^{-1}$ and $\Omega_{r 0} \approx 8 \times$ $10^{-5}$, the imposition that $L_{b}$ should be a few orders of magnitude bigger than the Planck length implies that $x_{b}<10^{31}$. Also, the bounce should occur before the beginning of nucleosynthesis, yielding $x_{b} \gg 10^{11}$. To summarize, one gets

$$
10^{11} \ll x_{b}<10^{31} .
$$




\section{Cosmological Perturbations in Quantum Bouncing Backgrounds}

In inflationary models, all wavelengths of cosmological interest were much smaller than the Hubble radius during the inflationary era, and any small perturbation around the homogeneous background would rapidly fade away. Hence, it is assumed that only quantum vacuum fluctuations would survive. This justifies the choice of an adiabatic vacuum state (close to the Bunch-Davies de Sitter vacuum state) as the initial quantum state of quantum cosmological perturbations, which is then evolved to the present era, with great observational success. In this scenario, the quantum perturbations evolve from the inflationary era up to now in a classical background, it is quantum field theory in curved spaces. However, the background classical cosmological models of inflation, when evolved back in time, generally contain an initial singularity, a point where no physics is possible, rendering them incomplete.

In the previous section, I have obtained quantum cosmological background models which are free of singularities. Furthermore, as explained in the Introduction, they contain a long-standing decelerating contracting phase, implying that they are also naturally free of the particle horizon and flatness issues. Finally, it was shown that the contracting phase can be smoothly connected through a quantum bounce to the usual radiation dominated expanding phase of the standard cosmological model. Hence, it is natural to extend the usual approach to cosmological perturbations to these models, in which one will have to consider quantum corrections to the background evolution itself. Note that, as in inflation, in bouncing models with decelerated contraction all wavelengths of cosmological interest are also much smaller than the Hubble radius in the far past of the contracting phase, which contains an immensely large space volume and very a small matter energy density, being very close to the flat Minkowski space-time. Hence, any small classical perturbation around such homogeneous background would rapidly dissipate away, and one can assume that only quantum vacuum fluctuations would survive (for details, see Reference [71]). Again, this justifies the choice of an adiabatic vacuum state as the initial quantum state of quantum cosmological perturbations, now close to the Minkowski vacuum quantum state, which should then be evolved to the present era. However, these quantum perturbations should be dynamically evolved through the bounce, which is not a trivial task, as long as the bounce usually involves new physics. This is an issue for many bouncing models. The situation is more complicate for quantum bounces, where the problem of interpretation of quantum cosmology becomes acute. Early attempts resulted in very complicate equations [72].

Nevertheless, if one applies the $\mathrm{dBB}$ quantum theory to quantum cosmology, then many of these issues can be naturally solved. As the background degrees of freedom and the perturbation fields are assumed to have physical reality with their own equations of motion (contrary to other approaches), the actual calculations are conceptually much simpler, because they are conceptually similar to the classical case. This point will become clear to the reader as we move on in this section, with concrete calculations (see References [73,74] for further details).

\subsection{Perfect Fluids}

Firstly, I will present the main features for the quantization of perturbations and background in the case of perfect hydrodynamical fluids $(p=w \rho)$.

The action we shall begin with is that of general relativity with a perfect fluid, the latter being described using the formalism of Section 2.2, yielding

$$
\mathcal{S}=\mathcal{S}_{\mathrm{GR}}+\mathcal{S}_{\text {fluid }}=-\frac{1}{2 l_{P}^{2}} \int \sqrt{-g} R \mathrm{~d}^{4} x-\int \sqrt{-g} p \mathrm{~d}^{4} x,
$$

remembering that $l_{P}=\left(8 \pi G_{N}\right)^{1 / 2}=\sqrt{6} \kappa$ is the Planck length in natural units, $\rho$ is the perfect fluid energy density satisfying the equation of state $p=\omega \rho$, where $p$ is the pressure and $\omega$ is a non-vanishing constant. 
Besides the usual homogeneous and isotropic cosmological background metric, restricted here to flat space-like hyper-surfaces,

$$
\mathrm{d} s^{2}=\bar{g}_{\mu \nu}(t) \mathrm{d} x^{\mu} \mathrm{d} x^{v}=N^{2}(t) \mathrm{d} t^{2}-a^{2}(t) \delta_{i j} \mathrm{~d} x^{i} \mathrm{~d} x^{j} .
$$

I will also consider linear scalar perturbations around it, $h_{\mu v}(t, \mathbf{x})$, which can be decomposed into

$$
\begin{aligned}
h_{00}(t, \mathbf{x}) & =2 N^{2}(t) \phi(t, \mathbf{x}) \\
h_{0 i}(t, \mathbf{x}) & =-N(t) a(t) B_{, i}(t, \mathbf{x}) \\
h_{i j}(t, \mathbf{x}) & \left.=2 a^{2}(t) \psi(t, \mathbf{x}) \gamma_{i j}-E_{, i j}(t, \mathbf{x})\right),
\end{aligned}
$$

where, $i$ represents $\partial / \partial x^{i}$. Hence, the total metric is

$$
g_{\mu v}(t, \mathbf{x})=\bar{g}_{\mu v}(t)+h_{\mu v}(t, \mathbf{x}) .
$$

The matter Lagrangian $p$ is expressed in terms of the fluid velocity field, see Section 2, which can be expanded in the same way,

$$
\varphi(t, \mathbf{x})=\bar{\varphi}(t)+\delta \varphi(t, \mathbf{x}),
$$

where $\bar{\varphi}(t)$ is the background field and $\delta \varphi(t, \mathbf{x})$ its first order perturbation.

One should also consider vector and tensor perturbations. The vector perturbations will be discussed in the next section. The tensor perturbations, or primordial gravitational waves, are represented by transverse-traceless spatial tensor $h_{i j}^{\mathrm{TT}}(t, \mathbf{x})$, whose treatment is very similar and much easier. For details, see References [73,75].

After substituting Equations (50), (51) and (53) into the Einstein-Hilbert action (49), one can construct the Hamiltonian of the system. The invariance of GR under diffeomorphisms induces the appearance of first class constraints, implying that there is only one degree of freedom describing the perturbations (see Reference [74] for details). After performing suitable canonical transformations, and without ever using the background equations of motion, the Hamiltonian up to second order can be reduced to

$$
H=N\left[H_{0}^{(0)}+H_{0}^{(2)}\right]
$$

where

$$
H_{0}^{(0)} \equiv-\frac{P_{a}^{2}}{4 a V}+\frac{P_{T}}{a^{3 \omega}}
$$

and

$$
H_{0}^{(2)} \equiv \frac{1}{2 a^{3}} \int \mathrm{d}^{3} x \pi^{2}(\mathbf{x})+\frac{a \omega}{2} \int \mathrm{d}^{3} x v^{, i}(\mathbf{x}) v_{, i}(\mathbf{x}) .
$$

Looking at (54), one can see that the final Hamiltonian contains a single first class constraint $H_{0}^{(0)}+H_{0}^{(2)}$, with Lagrange multiplier $N$, connected with the invariance of the theory under time reparametrizations. One recognizes $H_{0}^{(0)}$ as the Hamiltonian describing the background FLRW model with perfect fluids, presented in Section 2. The second order piece, $H_{0}^{(2)}$, describes the dynamics of the perturbation field, in which $v(\mathbf{x})$, with its conjugate momentum $\pi(\mathbf{x})$, emerges as the single perturbation degree of freedom left. It is the usual gauge invariant Mukhanov-Sasaki variable

$$
v(\mathbf{x})=\frac{a^{\frac{1}{2}(3 w-1)}}{\sqrt{6}}\left(\delta \varphi(\mathbf{x})+\frac{2 \sqrt{6} \sqrt{(w+1) P_{T}}}{P_{a} \sqrt{w}} a^{2-3 w} \psi(\mathbf{x})\right),
$$


written in terms of the background variables, and the perturbation fields $\psi(\mathbf{x}), \delta \varphi(\mathbf{x})$. The $v(\mathbf{x})$ field is connected to the gauge invariant Bardeen potential $\Phi(\mathbf{x})$ (see Reference [76]) through

$$
\Phi^{, i}, i(\mathbf{x})=-\frac{3 \sqrt{(\omega+1) \bar{\rho}}}{2 \sqrt{\omega}} a\left(\frac{v(\mathbf{x})}{a}\right)^{\prime},
$$

where $\bar{\rho}$ is the background energy density, and a prime means derivative with respect to conformal time.

In the Dirac quantization procedure, the first class constraints must annihilate the wave functional $\Psi[T, a, v(\mathbf{x})]$,

$$
\left(\hat{H}_{0}^{(0)}+\hat{H}_{0}^{(2)}\right) \Psi=0 .
$$

As $P_{T}$ appears linearly in $H$, and making the gauge choice $N=a^{3 w}$, one can interpret the $T$ variable as a time parameter. Hence, Equation (59) assumes the Schrödinger form

$$
\begin{aligned}
i \frac{\partial}{\partial T} \Psi & =\frac{1}{4}\left\{a^{(3 w-1) / 2} \frac{\partial}{\partial a}\left[a^{(3 w-1) / 2} \frac{\partial}{\partial a}\right]\right\} \Psi \\
& -\left[\frac{a^{3 w-1}}{2} \int d^{3} x \frac{\delta^{2}}{\delta v^{2}(\mathbf{x})}-\frac{a^{3 w+1} w}{2} \int d^{3} x v^{i}(\mathbf{x}) v, i(\mathbf{x})\right] \Psi,
\end{aligned}
$$

where we have chosen the same factor ordering in $a$ as in the previous section.

If one makes the ansatz

$$
\Psi[a, T, v(\mathbf{x})]=\Psi_{(0)}(a, T) \Psi_{(2)}[T, v(\mathbf{x})],
$$

which is based on an assumption that the background is not entangled with the perturbations, then at zeroth order one finds that $\Psi_{(0)}(a, T)$ satisfies the equation,

$$
\begin{aligned}
& i \frac{\partial}{\partial T} \Psi_{(0)}(a, T)= \\
& \frac{1}{4}\left\{a^{(3 w-1) / 2} \frac{\partial}{\partial a}\left[a^{(3 w-1) / 2} \frac{\partial}{\partial a}\right]\right\} \Psi_{(0)}(a, T),
\end{aligned}
$$

while $\Psi_{(2)}[v(\mathbf{x}), T]$ satisfies the equation

$$
\begin{aligned}
& i \frac{\partial}{\partial T} \Psi_{(2)}[T, v(\mathbf{x})]=-\frac{a^{(3 w-1)}}{2} \int d^{3} x \frac{\delta^{2}}{\delta v^{2}(\mathbf{x})} \Psi_{(2)}[T, v(\mathbf{x})]+ \\
& \frac{w a^{(3 w+1)}}{2} \int d^{3} x v^{i}(\mathbf{x}) v_{, i}(\mathbf{x}) \Psi_{(2)}[T, v(\mathbf{x})] .
\end{aligned}
$$

At this point, one can see an advantage in using the dBB theory. Solutions of the zeroth order equation yield, in the dBB theory, a Bohmian trajectory $a(T)$ which, when inserted in Equation (63), yields a time-dependent Schrödinger equation for $\Psi_{(2)}$, because $a(T)$ can be viewed as a given function of time. Then, going to conformal time $d \eta=a^{3 w-1} d T$, and performing the time-dependent unitary transformation

$$
U=\exp \left\{i \int d^{3} x\left[\frac{a(T) v(\mathbf{x})}{2 a(T)}-\frac{(v(\mathbf{x}) \pi(\mathbf{x})+\pi(\mathbf{x}) v(\mathbf{x}))}{2} \ln (a(T))\right]\right\},
$$

where $a(T)$ is the Bohmian trajectory, the functional Schrödinger equation for the perturbations transforms to

$$
i \frac{\partial \Psi_{(2)}[v(\mathbf{x}), \eta]}{\partial \eta}=\int \mathrm{d}^{3} x\left(-\frac{1}{2} \frac{\delta^{2}}{\delta v^{2}(\mathbf{x})}+\frac{w}{2} v_{, i}(\mathbf{x}) v^{\prime i}(\mathbf{x})-\frac{a^{\prime \prime}}{2 a} v^{2}(\mathbf{x})\right) \Psi_{(2)}[v(\mathbf{x}), \eta],
$$


where we have gone to the new quantum variable $\bar{v}(\mathbf{x})=a v(\mathbf{x})$, the Mukhanov-Sasaki variable defined in Reference [76] (we have omitted the bars). Equation (65) is the usual functional Schrödinger equation for quantum linear perturbations in cosmological models with a single perfect fluid. The difference with the conventional approach is that now the scale factor appearing in Equation (65) is not the classical one, but the Bohmian solution $a(\eta)$, which may be free of singularities, with a bounce. This interpretation of Equation (65) is only possible within the $\mathrm{dBB}$ theory, in which a Bohmian trajectory $a(\eta)$ can be defined. In other frameworks, where $a$ is a background quantum degree of freedom, hence a quantum operator, the physical understanding of the Wheeler-DeWitt equation (60) using the ansatz (61) implying Equation (65) becomes conceptually much more intricate, if possible. Solutions Equation (62) are known, and were presented in the previous section.

In the Heisenberg picture the operator $\hat{v}(\mathbf{x})$ evolves as

$$
\hat{v}^{\prime \prime}(\mathbf{x})-\omega \hat{v}^{i}{ }_{i}^{i}(\mathbf{x})-\frac{a^{\prime \prime}}{a} \hat{v}(\mathbf{x})=0 .
$$

In terms of the normal modes $v_{k}$, the above equation reads

$$
v_{k}^{\prime \prime}+\left(\omega k^{2}-\frac{a^{\prime \prime}}{a}\right) v_{k}=0 .
$$

These equations have the same form as the equations for scalar perturbations obtained in Reference [76]. However, as the function $a(\eta)$ is no longer a classical solution of the background equations, but a quantum Bohmian trajectory of the quantized background, different power spectra of quantum perturbations may arise.

Far from the bounce, when $|T| \gg\left|T_{b}\right|$, Equation (67) reads,

$$
v_{k}^{\prime \prime}+\left[\omega k^{2}+\frac{2(3 \omega-1)}{(1+3 \omega)^{2} \eta^{2}}\right] v_{k}=0
$$

The solution is

$$
v_{k}=\sqrt{|\eta|}\left[c_{1}(k) H_{v}^{(1)}(\bar{k}|\eta|)+c_{2}(k) H_{v}^{(2)}(\bar{k}|\eta|)\right],
$$

with

$$
v=\frac{3(1-\omega)}{2(3 \omega+1)},
$$

where $H^{(1,2)}$ are Hankel functions, $\bar{k} \equiv \sqrt{\omega} k$, and we are considering the far past of the contracting phase, $\eta \ll-1$.

Perturbation information propagates with the speed of sound $c_{s}=\sqrt{w}$, hence the scale of causal contact is the sound horizon $c_{s} R_{H}=\sqrt{w} a^{2} / a^{\prime}$. This must be compared with the perturbation scale, $\lambda a=a / k$. The bounce solution (45) for $|\eta| \gg \eta_{b}$ is a power low in $\eta$, hence these scales become comparable when $c_{s} R_{H} \approx \lambda a \Rightarrow \bar{k}|\eta| \approx 1$. Consequently, in the far past of the contracting phase, $\eta \ll-1$, one has $\bar{k}|\eta| \gg 1 \Rightarrow c_{s} R_{H} \gg \lambda a$, and all inhomogeneous perturbations of cosmological interest were far inside the sound Hubble radius, as anticipated in the Introduction, allowing a causal explanation for their origin, like in inflation. Furthermore, the Universe was almost empty and flat in this era (the space-time curvature behaves as $1 / \eta^{2} \rightarrow 0$ ), hence, small classical inhomogeneities would rapidly dissipate away [71], and only quantum vacuum fluctuations around the background homogeneity should be present. As a consequence, we will set as the initial quantum state of cosmological perturbations at this era the (almost) Minkowski vacuum state, with modes given by,

$$
v_{k}^{(\mathrm{ini})}=\frac{\exp i \bar{k} \eta}{\sqrt{\bar{k}}}
$$


This implies that

$$
c_{1}=0 \quad \text { and } \quad c_{2}=l_{P} \sqrt{\frac{3 \pi}{2}} \exp ^{-i \frac{\pi}{2}\left(v+\frac{1}{2}\right)} .
$$

In order to propagate the solution through the bounce up to the expanding phase, one can expand the solutions of Equation (68) in powers of $k^{2}$ according to the formal solution [76]

$$
\begin{aligned}
\frac{v_{k}}{a} & =A_{1}(k)\left[1-\omega k^{2} \int^{t} \frac{d \bar{\eta}}{a^{2}(\bar{\eta})} \int^{\bar{\eta}} a^{2}(\overline{\bar{\eta}}) d \overline{\bar{\eta}}\right] \\
& +A_{2}(k)\left[\int^{\eta} \frac{d \bar{\eta}}{a^{2}}-\omega k^{2} \int^{\eta} \frac{d \bar{\eta}}{a^{2}} \int^{\bar{\eta}} a^{2} d \overline{\bar{\eta}} \int^{\overline{\bar{\eta}}} \frac{d \overline{\bar{\eta}}}{a^{2}}\right]+\ldots,
\end{aligned}
$$

where I have omitted the terms of order $\mathcal{O}\left(k^{j \geq 4}\right)$. In Equation (71), the coefficients $A_{1}$ and $A_{2}$ are two constants depending only on the wavenumber $k$ through the initial conditions. Although Equation (71) should formally apply for all times, this presentation of the solutions can be very useful in the regions were $\bar{k} \eta \ll 1$, where terms of higher order $k$ become negligible. In order to find the $k$ dependence of $A_{1}$ and $A_{2}$, one can match solution (71) with Equation (69), where $c_{1}$ and $c_{2}$ are already known, yielding

$$
\begin{aligned}
& A_{1} \propto\left(\frac{\bar{k}}{k_{0}}\right)^{\frac{3(1-\omega)}{2(3 \omega+1)}} \\
& A_{2} \propto\left(\frac{\bar{k}}{k_{0}}\right)^{\frac{3(\omega-1)}{2(3 \omega+1)}}
\end{aligned}
$$

where $k_{0}^{-1}=T_{0} a_{0}^{3 \omega-1}=L_{b}$, and $L_{b}$ is the curvature scale at the bounce. One can then propagate solution (71) up to the expanding phase, and then find the power spectrum of primordial cosmological perturbations.

The Bardeen potential $\Phi(\mathbf{x})$ can be obtained from $v(\mathbf{x})$ through the formula

$$
\Phi^{, i}{ }_{i}(\mathbf{x})=-\frac{3 l_{P}^{2} \sqrt{(\omega+1) \bar{\rho}}}{2 \sqrt{\omega}} a\left(\frac{v(\mathbf{x})}{a}\right)^{\prime} .
$$

In the expanding phase, when the quantum effects allowing the bounce become negligible again, $T \gg T_{b}$, the constant mode of $\Phi$, like $v$, mixes $A_{1}$ with $A_{2}$. In this region, taking into account that $A_{2}$ dominates over $A_{1}$ (see the next section for details), one obtains, for the modes,

$$
\Phi_{k} \propto k^{\frac{3(\omega-1)}{2(3 \omega+1)}}\left[\text { const. }+\frac{1}{\eta^{(5+3 \omega) /(1+3 \omega)}}\right] .
$$

The power spectrum of the Bardeen potential

$$
\mathcal{P}_{\Phi} \equiv \frac{2 k^{3}}{\pi^{2}}\left|\Phi_{k}\right|^{2}
$$

reads

$$
\mathcal{P}_{\Phi} \propto k^{n_{S}-1}
$$

where

$$
n_{\mathrm{S}}=1+\frac{12 \omega}{1+3 \omega} .
$$


For gravitational waves (see Reference [75] for details), the equation for the modes $\mu_{k}=a h_{k}\left(h_{k}\right.$ is the amplitude of the waves) reads

$$
\mu_{k}^{\prime \prime}+\left(k^{2}-\frac{a^{\prime \prime}}{a}\right) \mu_{k}=0
$$

The power spectrum is

$$
\mathcal{P}_{h} \equiv \frac{2 k^{3}}{\pi^{2}}\left|\frac{\mu_{k}}{a}\right|^{2} \propto k^{n_{\mathrm{T}}}
$$

In Reference [75], we have obtained, as for the scalar modes,

$$
n_{\mathrm{T}}=\frac{12 \omega}{1+3 \omega}
$$

In the limit $\omega \rightarrow 0$ (dust), we obtain a scale invariant spectrum for both tensor and scalar perturbations. This result was confirmed through numerical calculations, which also gave the amplitudes [74]. In fact, it is a general result: in a smooth bounce, any contracting dust-dominated phase at large scales leads to an almost scale invariant spectrum for large wave-lengths scalar perturbations [77]. Note that it is not necessary that the fluid that dominates during the bounce be dust. The $k$-dependence of $A_{1}$ and $A_{2}$ is obtained far from the bounce, when the modes cross the sound Hubble radius, $\bar{k} \eta \approx 1$, and they do not change in a possible transition from matter to radiation domination in the contracting phase, or during the bounce. The effect of the bounce is essentially to mix these two coefficients, when the constant mode acquires the scale invariant piece. Hence, the bounce itself may be dominated by another fluid, like radiation.

Note that the model considered here, with one single perfect fluid, is still too simple, but it reveals that a dominance of a dust fluid in the far past of the contracting phase is essential, in the same way that the inflation should behave almost as a cosmological constant during the inflationary era. Of course the model must be more elaborated, as with a single perfect fluid one usually has $w=c_{s}^{2}>0$, and it will be hard to accommodate result (78) with a red-tilted spectrum, as observed. And indeed, just by including radiation in the model, a red-tilted spectral index can emerge. In fact, the more complex bounce solution (46) yields an almost scale invariant spectrum of adiabatic cosmological perturbations, with amplitude given by $[78,79]$,

$$
A_{S} \approx 10^{-2} \frac{l_{p}^{2}}{R_{H_{0}}^{2}} \frac{x_{b}^{2}}{\Omega_{r 0} c_{S}^{5}},
$$

where $c_{S}$ is the value of the sound velocity of the adiabatic perturbation when it crosses the sound Hubble radius. As the perturbation modes grow faster after they cross the sound Hubble radius, and as they cross earlier this scale for smaller $c_{S}, c_{S} k\left|\eta_{c}\right| \approx 1$, where $\eta_{c}$ is the conformal time at crossing (which is negative, as we are in the contracting phase), then the amplitude of scalar perturbations must increase inversely with $c_{S}$, as it is the case of Equation (82). As $A_{S} \approx 2.09 \times 10^{-9}$, see Reference [23], and using Equation (48), one gets

$$
10^{-16} \leq c_{S}<10^{-10}
$$

for the sound velocity. Note, however, that there are two fluids, hence the sound velocity for the adiabatic perturbations reads:

$$
c_{s}^{2}=\frac{w\left(\rho_{d}+p_{d}\right)+\left(\rho_{r}+p_{r}\right) / 3}{\rho_{T}+p_{T}},
$$

where $w$ is the equation of state parameter of the dust fluid, and the indices $d, r, T$ refers to dust, radiation, and total energy densities and pressures, respectively. Hence, $c_{S}^{2} \approx$ $|w| \ll 1$ only when the dust-matter fluid dominates, which is the case of the larger scales of cosmological interest. Smaller scales, or larger $k$ perturbation modes, cross the sound 
Hubble radius later, when radiation begins to be important, hence the power spectrum amplitude gets suppressed because $c_{S}$ increases up to the limit $1 / \sqrt{3}$ when radiation dominates, see Equation (84). Accordingly, the spectrum must be slightly red-tilted due to the presence of radiation, and the parameters may be fitted with CMBR observations.

Note that tensor perturbations have $c_{s}=1$, and as they evolve similarly to scalar perturbations, see Equation (79), their amplitudes must be very small in comparison with scalar perturbations in the case of bouncing models with fluids, being unobservable at large scales, or very small frequencies. I will return to this subject by the end of this section, discussing the large frequency regime.

In the next sections I will discuss other quantum bouncing models in which one can have $0.96<n_{s}<0.97$, either by considering dark energy, or by modeling the dust component by a canonical scalar field, in which the effective $w$ is not constrained to be positive, as it has no relation with the sound speed of adiabatic perturbations (which is 1 in this case).

\subsection{The Scalar Field}

As we have seen in the previous section, in the case of scalar fields there is no momentum which appears linearly in the Hamiltonian constraint, and the Wheeler-DeWitt equation has rather a Klein-Gordon form than a Schrödinger-like structure. Hence, the route to obtain a functional Schrödinger equation for the perturbations is more subtle than in the perfect fluid case. The aim of this subsection is to present the steps leading to a functional Schrödinger equation for the perturbations in the scalar field case. We will see that the $\mathrm{dBB}$ theory turns out to be a very useful tool to achieve this goal. In order to emphasize the concepts rather than technicalities, I will consider a massless free scalar field. The generalization to arbitrary potentials (like inflationary ones see Reference [80]) is straightforward.

The free massless scalar field is $\varphi(t, \mathbf{x})=\varphi(t)+\delta \varphi(t, \mathbf{x})$, where $\varphi(t)$ is the background homogeneous scalar field and $\delta \varphi(t, \mathbf{x})$ is its linear perturbation. The FLRW metric together with their scalar perturbations are given by Equations (52), (50) and (51).

Starting from the classical action for this system, the Hamiltonian up to secondorder can be brought into the following simple form, without ever using the background equations of motion [80] $\left(\kappa^{2}=1\right)$ :

$$
H=\frac{N}{2 e^{3 \alpha}}\left[-P_{\alpha}^{2}+P_{\varphi}^{2}+\int d^{3} x\left(\pi^{2}(\mathbf{x})+e^{4 \alpha} v^{\prime}(\mathbf{x}) v_{, i}(\mathbf{x})\right)\right],
$$

where again $a=\mathrm{e}^{\alpha}$ and $v(\mathbf{x})$ is the usual Mukhanov-Sasaki variable [76], defined as

$$
v(\mathbf{x})=a\left(\delta \varphi(\mathbf{x})+\frac{\varphi^{\prime} \phi(\mathbf{x})}{\mathcal{H}}\right),
$$

with primes denoting derivatives with respect to conformal time $\eta$, and $\mathcal{H}=a^{\prime} / a=\alpha^{\prime}$.

The Dirac quantization procedure yields the Wheeler-DeWitt equation

$$
\left(\hat{H}_{0}+\hat{H}_{2}\right) \Psi=0,
$$

where

$$
\begin{aligned}
& \hat{H}_{0}=-\frac{\hat{P}_{\alpha}^{2}}{2}+\frac{\hat{P}_{\varphi}^{2}}{2} \\
& \hat{H}_{2}=\frac{1}{2} \int d^{3} x\left(\hat{\pi}^{2}(\mathbf{x})+e^{4 \hat{\alpha}} \hat{v}^{, i}(\mathbf{x}) \hat{v}_{, i}(\mathbf{x})\right) .
\end{aligned}
$$

As in the perfect fluid case, I will suppose that the background evolution is not affected by the quantum perturbations through some quantum entanglement, yielding

$$
\Psi[\alpha, \varphi, v(\mathbf{x})]=\Psi_{0}(\alpha, \varphi) \Psi_{2}[\alpha, \varphi, v(\mathbf{x})]
$$


Here, I am letting $\Psi_{2}$ depend on both $\alpha, \varphi$, as there is no explicit background variable playing the role of time. As before, the zeroth order part Equation (87) reads

$$
\hat{H}_{0} \Psi_{0}=0
$$

and, in the $\mathrm{dBB}$ framework, the corresponding background guidance equations. This is the mini-superspace model described in Section 2. As we have seen, quantum effects can eliminate the background singularity, leading to bouncing models.

One can now proceed to the second order equation substituting the ansatz (90) into Equation (87) and using (91). Taking a Bohmian solution $(\alpha(t), \varphi(t))$ for the background, guided by $\Psi_{0}$, one can now use the Section 2 concept of conditional wave equation for the perturbations defining

$$
\chi[v(\mathbf{x}), t]=\Psi_{2}[\alpha(t), \varphi(t), v(\mathbf{x})] .
$$

As the dBB background quantization yields the guidance Equation (19) (remember that we are in the time gauge $N=e^{3 \alpha}$ and using $\kappa=1$ )

$$
\dot{\varphi}=\partial_{\varphi} S, \quad \dot{\alpha}=-\partial_{\alpha} S,
$$

then one can state that

$$
-\left(\frac{\partial S_{0}}{\partial \alpha}\right)\left(\frac{\partial \Psi_{2}}{\partial \alpha}\right)+\left(\frac{\partial S_{0}}{\partial \varphi}\right)\left(\frac{\partial \Psi_{2}}{\partial \varphi}\right)=\dot{\alpha}\left(\frac{\partial \Psi_{2}}{\partial \alpha}\right)+\dot{\varphi}\left(\frac{\partial \Psi_{2}}{\partial \varphi}\right)=\frac{\partial \chi}{\partial t} .
$$

Using Equation (94) in (87), together with two other assumptions which I will discuss shortly, one gets the Schrödinger equation

$$
i \frac{\partial \chi}{\partial t}=\hat{H}_{2} \chi,
$$

where, as before, the dependence of $\hat{H}_{2}$ on the background variables are understood as a dependence on $t$.

In order to go to conformal time, one can implement a time-dependent canonical transformation, similar to what was done in the perfect fluid case, yielding (see Reference [81] for details)

$$
i \frac{\partial \chi}{\partial \eta}=\frac{1}{2} \int d^{3} x\left[\hat{\pi}^{2}(\mathbf{x})+\hat{v}^{i}(\mathbf{x}) \hat{v}_{, i}(\mathbf{x})-\frac{a^{\prime \prime}}{a} \sqrt{\gamma} \hat{v}^{2}(\mathbf{x})\right] \chi,
$$

implying the mode equation

$$
v_{k}^{\prime \prime}+\left(k^{2}-\frac{a^{\prime \prime}}{a}\right) v_{k}=0
$$

Remember that we have not used the background equations of motion. Thus we have shown that Equation (97) is well defined, independently of the background dynamics, and it is correct even if we consider quantum background trajectories. Note that the time dependent potential $a^{\prime \prime} / a$ in Equation (97) can be rather different from the usual semi-classical calculations, because it is calculated from Bohmian trajectories, not from the classical ones. This can give rise to different effects in the region where the quantum effects on the background are important, which can propagate to the classical region.

Equation (96) was obtained under two conditions. The first is that, when the background gets near the classical limit one should obtain the usual semi-classical Schrödinger equation for the perturbations, without any further corrections. Indeed, there could be corrections originated from some quantum entanglement between the background and the perturbations affecting the quantum perturbations, even when the background is already classical, which would spoil the usual semi-classical approximation. This could be a viable possibility driven by a different type of wave functional than the one considered here, but it seems that our Universe is not so complicate. In fact, the observation that the simple 
semi-classical model without this sort of entanglement works well in the real Universe indicates something about the wave functional of the Universe. In other words, the validity of the usual semiclassical approximation imposes this first condition. One could also have some quantum entanglement effect on the perturbations from the background degrees of freedom when the background is fully quantum, and the final quantum Equation (96) for the perturbations we have obtained would not be valid around the quantum bounce. In this case, there is no observation that could guide us in the choice of the class of wave functionals that should be used. Hence, this choice resides only on assumptions of simplicity. This is our second condition. The mathematical expression for them can be found in Reference [81].

One can state the two conditions in a single form: there is no quantum entanglement effect of the background on the perturbations in the whole history of the Universe. This is the physical reason for choosing the specific class of wave functionals satisfying Equation (96). Taking other classes of wave functionals, which would not satisfy this physical hypothesis, can lead to new effects. This could also be an alternative interesting line of investigation.

I would like to emphasize that the original Wheeler-DeWitt equation has no time in it, but I was able to construct a Schrödinger equation for the perturbations. The assumption of the existence of a Bohmian background quantum trajectory was essential to achieve this goal, see Equation (94). This is very important, because a notion of probability emerges naturally in quantum equations for the wave functional with a Shrödinger form, but not with a Klein-Gordon form, which is the case of the original Wheeler-DeWitt equation. However, in the $\mathrm{dBB}$ approach no probability measure is required a priory, but this does not forbid us to obtain the Bohmian trajectories for the background through the guidance relations. These trajectories, which are assumed to be actual trajectories, can then be used in the equations for the perturbations, yielding a Schrödinger equation for them. In this case, there is a typical probability distribution, the Born distribution, which can be the attractor, called quantum equilibrium distribution (see Reference [58]), of any reasonable probability distribution. Hence, we are back to standard quantum theory of cosmological perturbations, now evolving in a background which does not always evolve classically and, thanks to that, is free of singularities.

When a scalar field potential is present, one just has to substitute $a^{\prime \prime} / a$ by $z^{\prime \prime} / z$ in this Hamiltonian, where $z=a \varphi^{\prime} / \mathcal{H}$, remembering that $z$ must be calculated using the Bohmian trajectories [80]. I will let to Section 5 the calculation of the power spectrum of a scalar field model with an exponential potential, because it will address some new issues that I will raise in the following sections.

\subsection{Gravitational Waves}

All models for the origin of primordial cosmological perturbations must be compared among themselves, and with observations, with the search for tests allowing to discriminate the models. One possible observational test can be through the detection of primordial gravitational waves (tensor perturbations). With this in mind, we have also calculated their amplitudes in the quantum bouncing models with perfect fluid discussed in Section 3 [82]. We have shown that the strain spectrum of the stochastic background of relic gravitons in such models is indeed quite different from the cyclic (ekpyrotic) and inflationary scenarios, see Figure 1. However, we have shown that the resulting amplitude is too small to be detected by any gravitational wave detector, unless in the frequency range $10-100 \mathrm{~Hz}$, as it can be seen from Figure 1. Nevertheless, it is a hard technical challenge to construct detectors of stochastic gravitational waves able to make significant observations in this range of frequencies. If it is possible, then one could use these observations to discriminate these primordial universe models, see Reference [82] for details. 


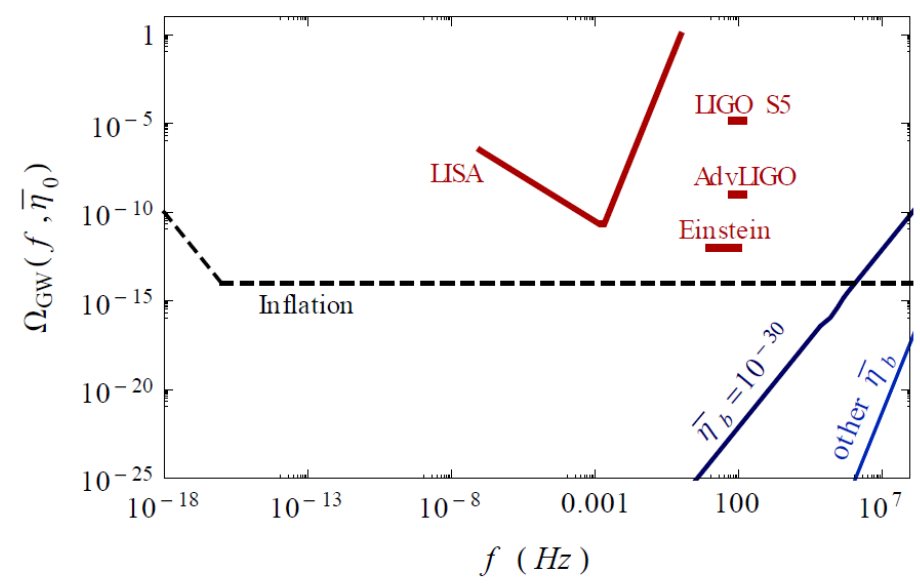

Figure 1. The figure shows a comparison of our results, labeled by $\bar{\eta}_{b}$ (the smaller this parameter the bigger is the energy scale of the bounce, and the value $10^{-30}$ is just two orders of magnitude away from the Planck scale) with experimental sensitivities of LIGO's 5th run, Advanced LIGO, and the forthcoming LISA and Einstein Telescope and a prediction of the upper limits on the spectrum of primordial gravitational waves generated in inflationary models.

In the scalar field case, the situation is different. In classical bounces, canonical scalar fields usually implies an amount of primordial gravitational waves comparable with scalar perturbations $[83,84]$, which is ruled out by observations. In Section 5 I will show how this problem is overcome in quantum bounces.

\section{Bounce Stability}

One objection against bouncing models concerns the non-linear growth of inhomogeneities during the contracting phase, implying that, after the bounce, in the beginning of the expanding phase, the Universe would be very far from the homogeneous configuration inferred by cosmological observations. Furthermore, a very inhomogeneous bounce would be very hard to describe, either through GR, or any of its extensions.

We argued in the Introduction that, up to now, there is no physical explanation for the initial homogeneity and isotropy of the Universe, either in a bouncing scenario or in a universe with a beginning followed by inflation. Hence, our approach to this issue was to assume from the beginning that the Universe was initially very homogeneous and isotropic, as observations impose to us. However, initial small inhomogeneous perturbations must be present in this homogeneous background, if not, there would be no structure in the Universe. The issue then is to evaluate whether these small perturbations would remain linear, at least up to the CMBR last scattering surface.

In a Universe with a beginning followed by inflationary expansion, one argues that only quantum vacuum fluctuations of these inhomogeneous perturbations survive. Then, it can easily be shown that they remain linear up to the necessary era when they cluster non-linearly to create structures in the Universe.

In bouncing models the situation can be more involved, as the Universe experiences a long era of contraction before the bounce, contrary to the accelerated expansion of the inflationary scenario. Intuitively, one might guess that such long contracting phase would favor a huge growth of these tiny inhomogeneous perturbations. In fact, this has always been a repeated claim against bouncing models.

In this section, I will show that the bouncing models discussed in this paper are stable, and their inhomogeneous perturbations remain linear up to the expanding phase when they should start to clump to form structures. As argued above, our point of departure will be an immensely large universe, with a tiny background homogeneous and isotropic matter energy density, hence being an almost flat space-time, where inhomogeneous perturbations arise from quantum vacuum fluctuations, as we explained in Section 3. I will investigate the evolution of scalar and vector perturbations, as the models we are considering already 
have tensor perturbations smaller then scalar perturbations in order to satisfy present observations [1].

\subsection{Scalar Perturbations}

It was shown in [85] that the curvature perturbation $\zeta=v / z$ is amplified in the contracting phase, and remains constant and scale invariant in the expanding phase. Nevertheless, the Bardeen potential $\Phi$ develops a large growing mode in the contracting phase, which is converted entirely into a decaying mode in the expanding phase, differently from inflation, where the decaying mode is always decreasing and small.

Even though the growing mode of $\Phi$ couples only with the decaying mode of the curvature perturbation in the expanding phase, the large value of the Bardeen potential at the bounce raises a problem concerning the violation of linear perturbation theory around this time, as long as in certain gauges it is directly connected to metric perturbations. In Reference [77] it was shown, using a specific model, that indeed the Bardeen potential grows larger than 1 at the bounce, but it can be made harmless with a suitable gauge choice. However, it remains the question about whether this problem is a general feature of bouncing models, breaking the homogeneity of space-time before the expanding phase, and, in case this is true, whether there exists efficient gauge choices which can solve this problem in a larger class of models. In order to address these questions, it was shown in Reference [79] that the ratio between the Bardeen potential at the bounce and its constant value after the bounce is a very large number in general. As this constant value is given by the CMBR observations, then the Bardeen potential is indeed very large at the bounce, putting into question the validity of perturbation theory around a homogeneous geometry around the bounce. In order to tackle this problem, it was found a set of necessary conditions that must be satisfied in a valid linear perturbation theory for the metric and matter perturbations. The first part of this set is defined by imposing that the metric perturbations remain small when compared to their background values. The second one comes through the imposition that the perturbed Einstein equations remain small when compared with the background dynamics. When these conditions are applied to the perturbations, it is necessary and sufficient that they should be satisfied at least in one specific gauge. In other words, one should have to find at least one way of embedding the reference gravitational and matter field configurations in the exact field configurations in such a way that the difference between both satisfies all established smallness conditions.

The calculation of the ratio between the Bardeen potential at the bounce, $\Phi_{b}$, with it expanding phase value when the decaying mode becomes negligible, $\Phi_{0}$, reads, for long wavelengths,

$$
\frac{\sqrt{\mathcal{P}_{\Phi}^{b}}}{\sqrt{\mathcal{P}_{\Phi}^{0}}}=\frac{\left|\Phi_{k}^{b}\right|}{\left|\Phi_{k}^{0}\right|}=\frac{\Omega_{0 w} 3\left(1-w_{q}\right)\left(5+3 w_{q}\right)}{4 \pi\left(\sqrt{w_{q}} k\right)^{2}} x_{b}^{1+3 w_{q}},
$$

where $\Omega_{0 w}=\rho_{0 w} / \rho_{c}, \rho_{c}$ is the critical density, $\rho_{0 w}$ is the energy density today of the fluid which dominates the contracting phase when the perturbation scale crosses the sound Hubble radius, which should satisfy $|w|=\left|p_{w} / \rho_{w}\right| \ll 1$ in order to yield an almost scale invariant spectrum of scalar perturbations, $w_{q}$ is the equation of state parameter of the fluid which dominates the quantum bounce, $x=a_{0} / a$, and $x_{b}=a_{0} / a_{b}$. As CMBR observations [1] require $\sqrt{\mathcal{P}_{\Phi}^{0}} \approx 10^{-5}$ and $x_{b} \gg 10^{11}$ (remember that the bounce must occur before nucleosynthesis, satisfying $x_{n} \approx 10^{11}$ ), then $\sqrt{\mathcal{P}_{\Phi}^{b}} \gg 1$. In the Newtonian gauge $\left(\mathcal{B}=0=\mathcal{E}\right.$, and consequently the shear $\left.\delta \sigma=-a\left(\mathcal{E}^{\prime}-\mathcal{B}\right)=0\right), \Phi=\phi$, which is the $g_{00}$ metric perturbation. Therefore linear perturbation theory breaks down in this gauge.

Note, however, that a valid gauge transformation relating different gauge fixations in some period of the cosmological history may not be valid in another era, because the gauge transformation parameter may become non-linear. Hence, one of the gauge fixations is not adequate in this period, and it does not mean that the perturbation theory breaks down, it only means that this particular gauge is not appropriate to describe the model 
evolution in this epoch. The correct approach is to verify that there exists at least one gauge choice in which the equations for the perturbations makes sense at linear order at each particular phase of the model history, before, of course, the usual epoch where non-linearities become important. This was done in Reference [79]. For the problem raised above, one can choose the constant curvature gauge $\left(\delta \mathcal{R}=-\frac{4}{a^{2}} D^{2} \psi=0\right.$, where $D^{2}$ is the spatial covariant Laplacian), in order to avoid the problem with $\phi$ described above. In this gauge one has $\psi=\mathcal{B}=0$, but one must impose $\mathcal{E}\left(\eta_{1}\right)=0$ at some convenient conformal time $\eta_{1}$ in order to fix the gauge completely. This gauge fixation yields

$$
\delta \sigma=-\frac{a \Phi}{\mathcal{H}}, \quad \phi=x^{2} c_{s}^{2} z^{2} \zeta, \quad \mathcal{E}=\int_{\eta_{1}}^{\eta} \frac{\mathrm{d} \bar{\eta} \overline{\mathcal{H}}}{\overline{\mathcal{H}}},
$$

where I used that $\Phi=\Psi$ (no anisotropic pressure is present, see Reference [79] for details).

One can note that, in this gauge, the metric perturbation $\phi$ has a different behavior with respect to $\zeta$. Indeed, when just a single fluid dominates, one gets

$$
\phi=\frac{3(1+w)}{2} \zeta
$$

In this gauge, $\phi$ is proportional to $\zeta$. As $\zeta$ grows in the contracting phase up to the bounce, when it acquires its maximum value and remains constant in the the expanding phase, and as it was proven for many bouncing models that this constant can be easily fitted with its observed value (which is small), then $\phi$ is always small as well. Hence, $\phi \ll 1$ is satisfied in this gauge.

There are other requirements which scalar perturbations should satisfy in order to keep linearity. They were analyzed in great detail in References [79,86], and they were shown to be satisfied all over the contracting phase and through the bounce itself. The conclusion was that, for a variety of models studied (not only the quantum bounce), the evolution of scalar perturbations near such bouncing models is well-behaved in what concerns the perturbative series.

\subsection{Vector Perturbations}

Another old question surrounding bouncing models concerns their stability under vector perturbations. Vector perturbations evolve kinematically as $a^{-2}$, when the matter content is described by perfect fluids or scalar fields. Consequently, a definite answer concerning the bounce stability depends on an arbitrary constant, hence, there is no definitive answer. In order to tackle this issue, it was considered a more general situation, where the primeval material medium is a non-ideal fluid, and its shear viscosity is capable of producing torque oscillations, which can create and dynamically sustain vector perturbations along cosmic evolution.

The total energy-momentum tensor considered was,

$$
T_{\mu v}=(\rho+p) u_{\mu} u_{v}-p g_{\mu v}+T_{\mu \nu}^{S},
$$

where $\rho$ is the fluid energy density, $p$ its pressure, $u^{\mu}$ is the four velocity, and $T_{S}^{\mu v}$ is the shear viscosity component of the fluid, given by

$$
T_{\mu v}^{S}=2 \lambda \sigma_{\mu v}
$$

where the shear reads

$$
\sigma_{\mu v} \equiv u_{(\mu ; v)}-u_{(\mu} u^{\rho} u_{v) ; \rho}-\frac{u^{\rho} ; \rho}{3}\left(g_{\mu \nu}-u_{\mu} u_{v}\right) .
$$


In this expression, $\lambda$ is the shear viscosity coefficient, ' $;$ ' the covariant derivative compatible with the metric, and $u^{\mu}$ is the normal vector orthogonal to the spatial hypersurfaces. Round brackets in the indices indicate symmetrization.

The background metric together with their vector perturbations is

$$
d s^{2}=a(\eta)^{2}\left[\mathrm{~d} \eta^{2}-\left(\delta_{i j}-\partial_{j} F_{i}(\mathbf{x})-\partial_{i} F_{j}(\mathbf{x})\right) \mathrm{d} x^{i} \mathrm{~d} x^{j}\right] .
$$

The linear perturbation $F_{i}(\mathbf{x})$ can be decomposed in terms of eigenfunctions of the three dimensional Laplace's operator, $Q_{i}(\mathbf{x})$, satisfying the equations,

$$
\begin{gathered}
\nabla^{2} Q_{i}(\mathbf{x})=-k^{2} Q_{i}(\mathbf{x}), \quad \partial_{i} Q^{i}(\mathbf{x})=0: \\
F_{i}^{(k)}=F_{k}(\eta) Q_{i} .
\end{gathered}
$$

The final mode equation for the perturbation $h_{k}(\eta)=-k F_{k}(\eta)$ reads (see Reference [87] for details)

$$
h_{k}^{\prime \prime}+\frac{2 a^{\prime}}{a} h_{k}^{\prime}+k^{2} b^{2} h_{k}=0,
$$

where $b^{2}=v_{t}^{2} / c^{2}$, with $v_{t}$ being the torsional velocity of sound.

In this framework, one can set that vector perturbations have a quantum mechanical origin, coming from quantum vacuum fluctuations in the far past of the bouncing model, as it is done with scalar and tensor perturbations (Equation (106) has the same structure as the equation for the tensor perturbation $h_{k}^{\mathrm{T}}=\mu_{k} / a$ in Section 3). Under this prescription, one can calculate their evolution during the whole history of the bouncing model, and precisely infer the conditions under which they remain linear before the expanding phase.

The bouncing model I will consider is the solution (46) presented in Section 2

$$
Y\left(\eta_{s}\right) \equiv \frac{a\left(\eta_{s}\right)}{a_{0}}=\frac{\Omega_{m 0}}{4} \eta_{s}^{2}+\sqrt{\frac{1}{x_{b}^{2}}+\Omega_{r 0} \eta_{s}^{2}},
$$

recalling that $a_{0}$ is scale factor today, $\Omega_{m 0}$ and $\Omega_{r 0}$ are the usual dimensionless densities of pressureless matter and radiation, respectively, $x_{b}=a_{0} / a_{b}, a_{b}$ being the value of the scale factor at the bounce, and $\eta_{s}=\left(a_{0} / R_{H_{0}}\right) \eta$.

In terms of the dimensionless variables,

$$
k_{s}=\frac{k R_{H_{0}}}{a_{0}},\left|h_{k_{s}}\right|=\sqrt{\frac{a_{0}^{3}}{16 \pi l_{p}^{2} R_{H_{0}}}}\left|h_{k}\right|,
$$

the conditions of linearity read (see Reference [87] for details)

$$
\left\langle h^{2}\right\rangle=\frac{8}{\pi} \frac{l_{p}^{2}}{R_{H_{0}}^{2}} \int_{k_{s, \min }}^{k_{s, \max }} \mathrm{d} k_{s} k_{s}^{2}\left|h_{k_{s}}\right|^{2} \ll 1,
$$

and

$$
\frac{4 \pi Y^{2} l_{p}^{2}}{\Omega_{r 0} R_{H_{0}}^{2}} \int_{k_{s, \min }}^{k_{s, \max }} \mathrm{d} k_{s} k_{s} P_{v}\left(k_{s}\right) \ll 1 .
$$

We have numerically checked the validity of these conditions. In the parameter space $\left(b, x_{b}\right)$ the allowed region of parameters in which conditions (109) and (110) are satisfied is shown in Figure 2: 


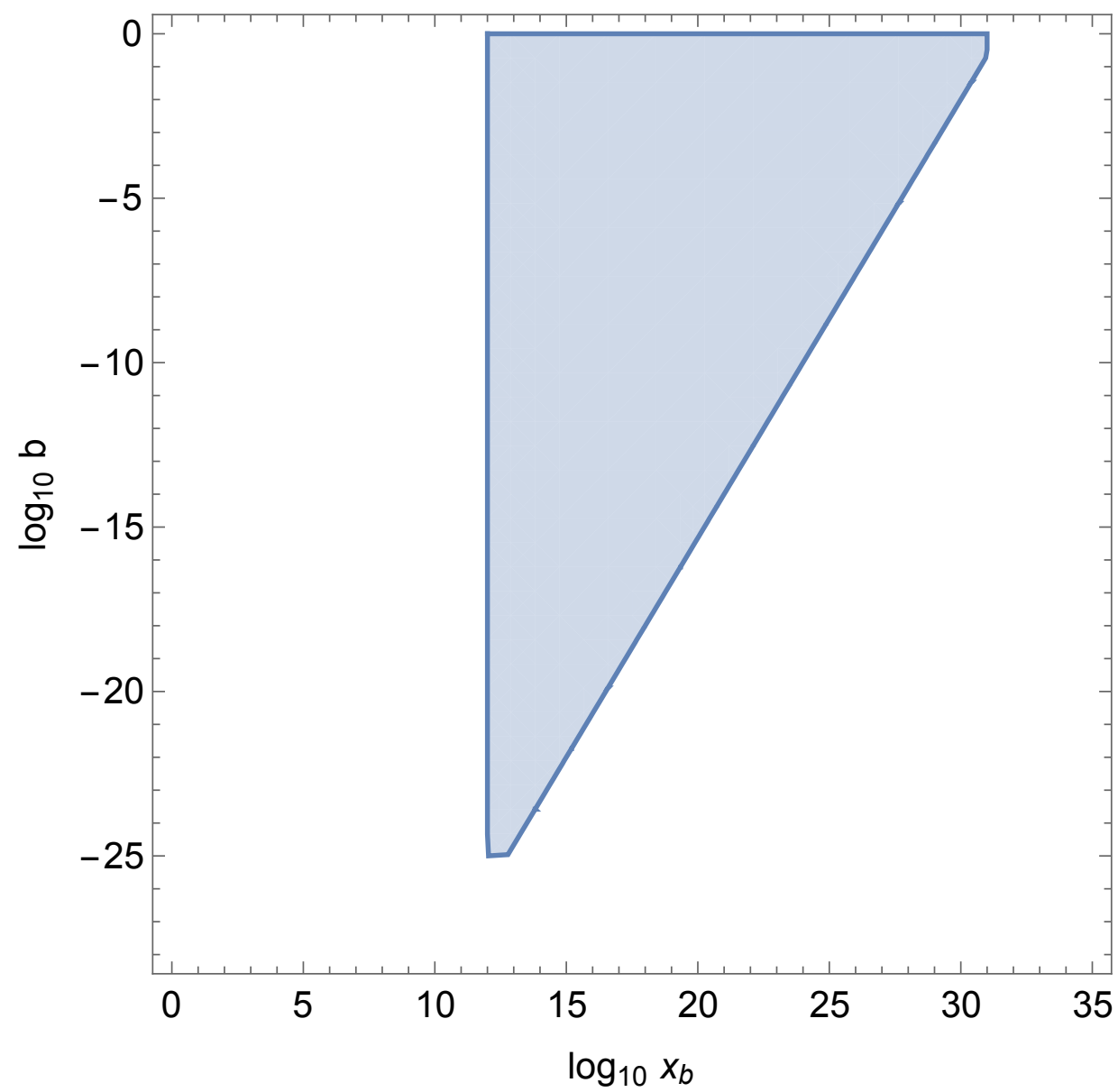

Figure 2. Region of stability under vector perturbations in parameter space for the bouncing models considered. The colored area represents the values of $b$ and $x_{b}$ which satisfies the consistency conditions (109) and (110).

Hence, there is a large range of parameters in which this model is stable under vector perturbations. Note that the minimum value allowed for $b$ is $b \approx 10^{-26}$. Such conditions impose that vector perturbations are also not observationally relevant in the expanding phase. The conclusion is that bouncing models are generally stable under vector perturbations. As they are also stable under scalar and tensor perturbations, we conclude that bouncing models are generally stable under perturbations originated from quantum vacuum perturbations in the far past of their contracting phase.

\section{The Presence of Dark Energy}

It has been observed that the present expansion of the Universe is accelerated [3,4], indicating the existence of a new energy component with negative pressure $p / \rho<-1 / 3$, probably a cosmological constant, called dark energy (DE). It is then natural to ask what is the role of DE in bouncing models. As we have seen, in bouncing models without DE, vacuum initial conditions for quantum cosmological perturbations are set in the far past of the contracting phase, when the Universe was very big and almost flat, justifying the choice of an adiabatic Minkowski vacuum in that phase. However, if DE exists and it dominates the Universe dynamics at large scales, it will also dominate the asymptotic past of bouncing models, modifying its geometric structure. As an important example, in the case of the standard $\Lambda \mathrm{CDM}$ model, where DE is a cosmological constant, the asymptotic past of such models approaches de Sitter rather than Minkowski geometry. Furthermore, the large wavelengths today become comparable with the Hubble radius in the contracting phase when the Universe was slightly influenced by the cosmological constant. Hence, the existence of a cosmological constant, or other types of DE, can modify 
the spectrum and amplitude of cosmological perturbations. Note that this is not a question for inflation, because initial conditions for quantum perturbations and the moment of Hubble radius crossing in such models take place at very small scales, when any DE causing the acceleration of the Universe today would be completely irrelevant. In fact, the presence of dark energy in the contracting phase of bouncing models may turn problematic the imposition of vacuum initial conditions for cosmological perturbations in the far past of such models.

In the case of a simple cosmological constant, all modes will eventually become larger than the curvature scale in the far past, and an adiabatic vacuum prescription becomes quite contrived, see Reference [88]. When a cosmological constant is present, a Minkowski adiabatic vacuum can only be defined in a definite period of time, from the end of cosmological constant domination, but when the Universe is still very big. Note, however, that the long wavelengths of physical interest today are not much smaller than the length scale associated with the value of cosmological constant given in the $\Lambda \mathrm{CDM}$ standard cosmological model, hence the spectrum of cosmological perturbations at these scales can be influenced by the presence of the cosmological constant. We have shown in Reference [88], analytically and numerically, that in a bouncing model containing a dust fluid, $w \approx 0$, an almost scale invariant spectrum of long wavelength perturbations is also obtained, but it is now affected by presence of the cosmological constant. It induces small oscillations, and a small running towards a red-tilted spectrum in these scales, see Figure 3. Hence, small oscillations in the spectrum of temperature fluctuations may arise in the cosmic background radiation at large scales, superimposed to the usual acoustic oscillations.

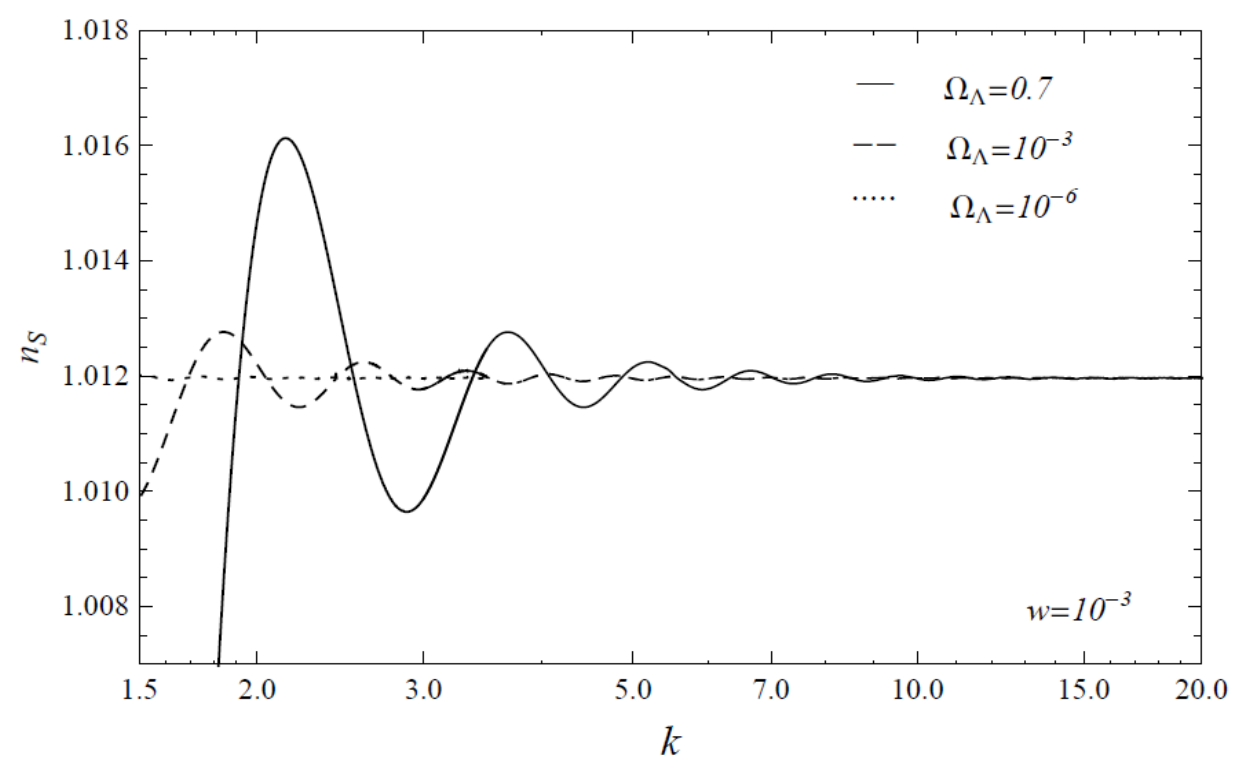

Figure 3. Numerical results for $n_{S}(k)$ in the presence of a cosmological constant. The solid line shows the result obtained using $\Omega_{\Lambda}=0.7$, the dashed line for $\Omega_{\Lambda}=10^{-3}$ and the dotted line for $\Omega_{\Lambda}=10^{-6}$. The oscillations become smaller for smaller $\Omega_{\Lambda}$, indicating that they arise because of the presence of the cosmological constant.

The case where DE is described by a scalar field was also investigated, see Ref. [89]. Consider $V_{G}=0$ and the exponential matter potential

$$
V_{M}(\varphi)=V_{0} \mathrm{e}^{-\lambda \bar{\kappa} \varphi},
$$

where $V_{0}$ and $\lambda$ are constant. $\bar{\kappa}^{2}=6 \kappa^{2}=8 \pi G$, so that $\lambda$ is dimensionless. Exponential potentials have been widely explored in cosmology in order to describe primordial inflation, the present acceleration of the universe, and matter bounces. This is because they contain 
attractor solutions, where the ratio between the pressure and the energy density is constant: $w=p / \rho$, where $w=\left(\lambda^{2}-3\right) / 3$. In order to describe matter bounces, $w \approx 0$, one sets $\lambda \approx \sqrt{3}$. I will now discuss in detail this case.

The classical dynamics of canonical scalar fields with exponential potential is very rich and simple to understand. Assuming the gauge $N=1$ (so that the time is cosmic proper time), one can define the variables

$$
x=\frac{\bar{\kappa}}{\sqrt{6} H} \dot{\varphi}, \quad y=\frac{\bar{\kappa} \sqrt{V_{M}}}{\sqrt{3} H},
$$

where

$$
H=\frac{\dot{a}}{a}=\dot{\alpha}
$$

is the Hubble parameter. This choice reduces the cosmological equations to the simple form

$$
\frac{\mathrm{d} x}{\mathrm{~d} \alpha}=-3\left(x-\frac{\lambda}{\sqrt{6}}\right)(1-x)(1+x)
$$

and the Friedmann equation to

$$
x^{2}+y^{2}=1
$$

The ratio $w=p / \rho$ is given by

$$
w=2 x^{2}-1
$$

As I am interested in studying matter bounces, I will set $\lambda=\sqrt{3}$.

The critical points are very easy to be identified from (114), and they are listed in Table 1, see Reference [90]. The critical points at $x= \pm 1$ with $w=1$ ( $p=\rho$, region the scalar field behaves as stiff matter) correspond to the space-time singularity $a=0$. Around this region, the potential is negligible with respect to the kinetic term. The critical points $x=1 / \sqrt{2}$ with $w=0$ ( $p=0$, the scalar field behaves as dust matter) are attractors (repellers) in the expanding (contracting) phase. Asymptotically in the infinite future (past), they correspond to very large slowly expanding (contracting) universes, and the space-time is asymptotically flat. Note that at $x=0$ the scalar field behaves like dark energy, $w=-1$, $p=-\rho$.

Table 1. Critical points of the planar system defined by (114) and (115).

\begin{tabular}{ccc}
\hline$x$ & $y$ & $w$ \\
\hline-1 & 0 & 1 \\
1 & 0 & 1 \\
$\frac{\lambda}{\sqrt{6}}$ & $-\sqrt{1-\frac{\lambda^{2}}{6}}$ & $\frac{1}{3}\left(\lambda^{2}-3\right)$ \\
$\frac{\lambda}{\sqrt{6}}$ & $\sqrt{1-\frac{\lambda^{2}}{6}}$ & $\frac{1}{3}\left(\lambda^{2}-3\right)$ \\
\hline
\end{tabular}

Consequently, we have four possible classical pictures:

(a) A classical dust contraction from very large scales, the initial repeller of the model, ending in a big crunch classical singularity around stiff matter domination with $x \approx 1$. The scalar field evolves smoothly from $w=0$ to $w=1$ behavior, without ever passing through a dark energy phase.

(b) A classical dust contraction from very large scales, the initial repeller of the model, ending in a big crunch classical singularity around stiff matter domination with $x \approx 1$. Now the scalar field passes through a dark energy phase, evolving smoothly from $w=0$ to $w=-1$ behavior, and then from $w=-1$ to $w=1$.

(c) The universe emerges from a classical Big Bang singularity around stiff matter domination with $x \approx 1, w=1$, and expands to an asymptotically dust matter domination phase, the attractor of the model. The scalar field evolves smoothly from $w=1$ 
to $w=0$ behavior, without ever passing through a dark energy phase. This is the time-reversed of case a.

(d) The universe emerges from a classical Big Bang singularity around stiff matter domination with $x \approx 1, w=1$, and expands to an asymptotically dust matter domination phase, the attractor of the model. Now the scalar field passes through a dark energy phase, evolving smoothly from $w=1$ to $w=-1$ behavior, and then from $w=-1$ to $w=0$. This is the time-reversed of case $b$.

These classical possibilities are shown in Figure 4. The trajectories take place on a circle. The points $M_{ \pm}$are respectively the dust attractor and repeller, while $S_{ \pm}$are the singularities: the upper semi-circle is disconnected from the down semi-circle, and they respectively describe the expanding and contracting solutions.

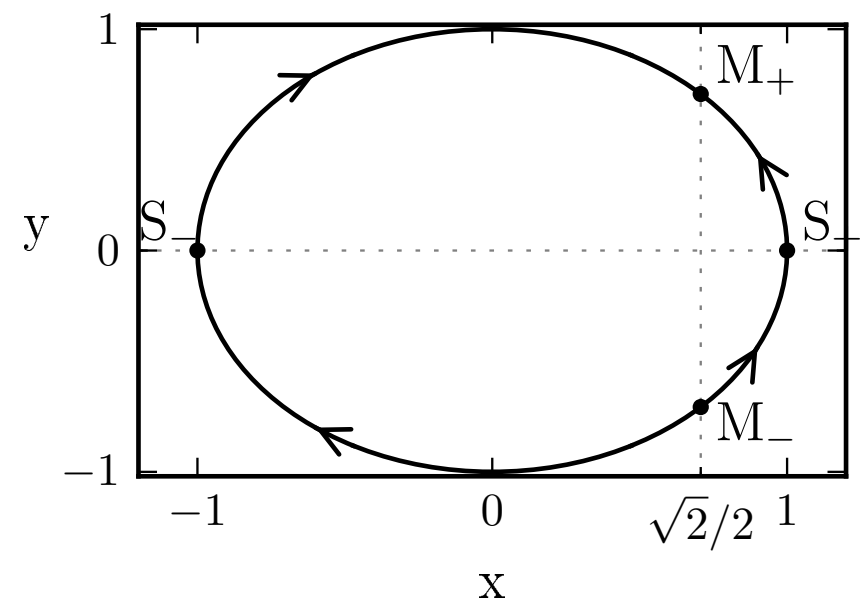

Figure 4. Phase space for the planar system defined by (114) and (115). The critical points are indicated by $M_{ \pm}$for a dust-type effective equation of state, and $S_{ \pm}$for a stiff-matter equation of state. Note that the region $y<0$ shows the contracting solutions, while the $y>0$ region presents the expanding solutions. Lower and upper quadrants are not physically connected, because there is a singularity in between.

In the quantum case, Bohmian bounce solutions were found. Exact solutions were given in Reference [91], and through matching conditions in Reference [89], yielding the same qualitative picture. In these solutions, the quantum effects are relevant near the singularity. In this region, the potential is negligible and the quantum bounce is similar to the ones described in the preceding section or as in [69], whose trajectories around the bounce are shown in Figure 5. For large scale factors, $\alpha>>1$, the classical stiff matter behavior is recovered, $x \approx \pm 1$, and from there on the Bohmian trajectory become classical, as described above. 


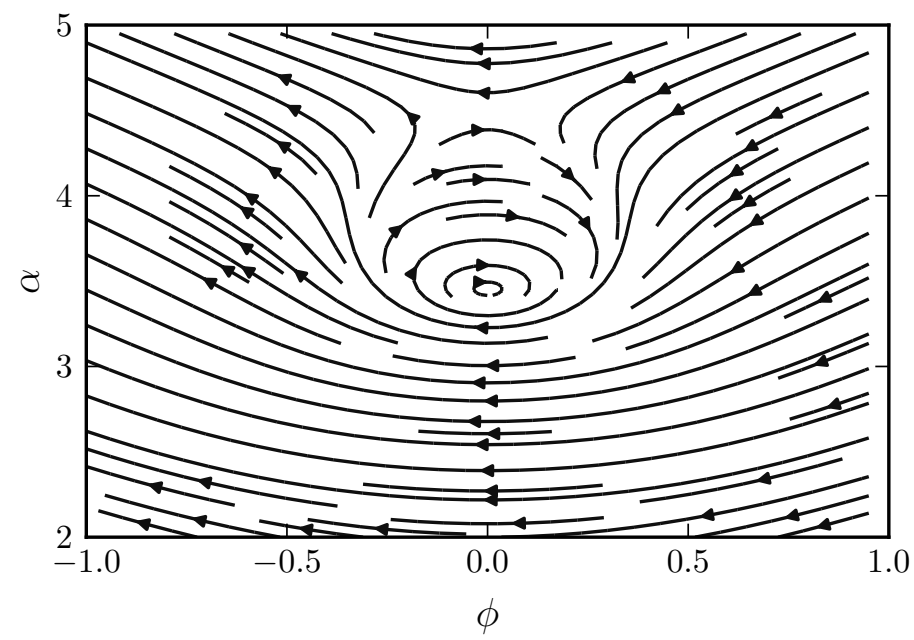

Figure 5. Phase space for the quantum bounce [89]. There are bounce and cyclic solutions. The bounces in the figure correspond to case B, where $\dot{\varphi}<0$. They connect regions around $S_{+}$in the contracting phase with regions around $S_{-}$in the expanding phase.

One very important observation is that, looking at Figure 5, the bounce can only connect $x \approx \pm 1$ classical stiff matter domination regions with $x \approx \mp 1$ classical stiff matter regions, respectively. In fact, a phase space analysis in terms of the $\mathrm{dBB}$ theory shows that this is the only way to connect the contracting solution to the expanding ones [89,91]. This fact implies that there are only two possible bouncing scenarios, see Figures 6 and 7:

(A) A classical dust contraction from very large scales, which passes through a dark energy phase up to a classical stiff matter contracting phase with $x \approx-1$. In this region, quantum effects become important, and a bounce takes place, launching the universe to a classical stiff matter expanding phase with $x \approx 1$, which then evolves to an asymptotically dust matter expanding phase, without passing through a dark energy phase.

(B) A classical dust contraction from very large scales directly to a classical stiff matter contracting phase with $x \approx 1$, without ever passing through a dark energy phase. In this region, quantum effects become relevant, and a bounce takes place, launching the universe to a classical stiff matter expanding phase with $x \approx-1$, and passing through a dark energy phase before reaching the asymptotically dust matter expanding phase.

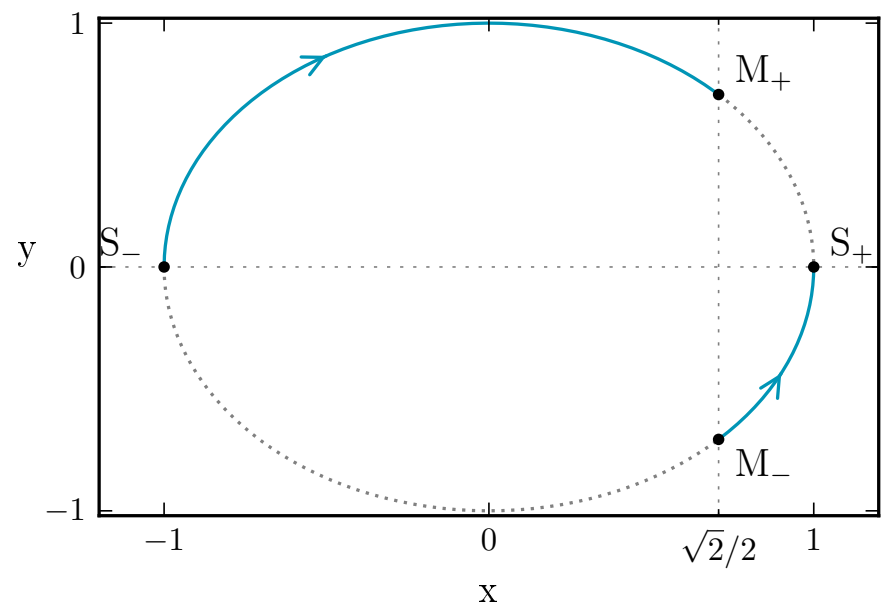

Figure 6. Case A: the scalar field has a dark energy phase during contraction. This model cannot describe the dark energy in the future, since the matter attractor is reached before. 


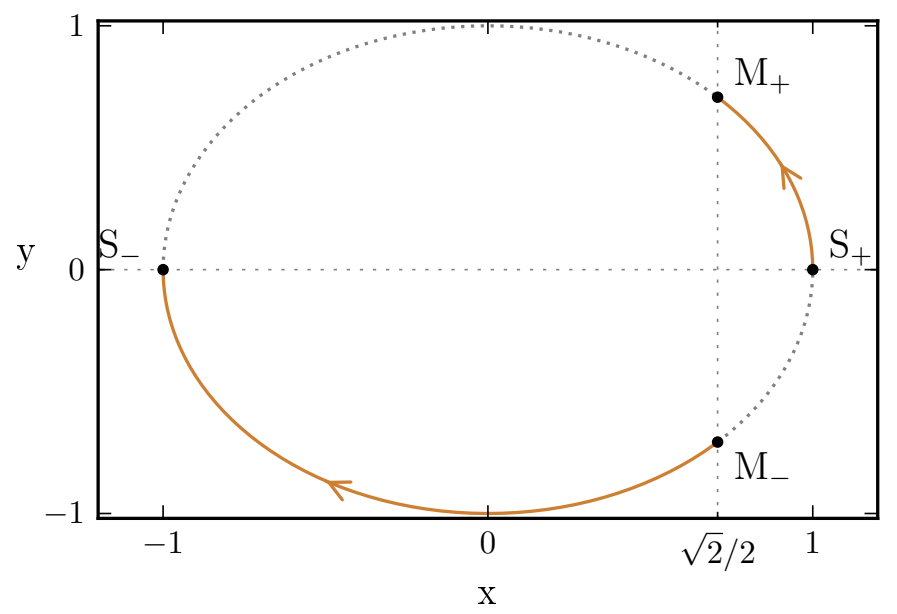

Figure 7. Case B: asymptotically dust contraction close to the unstable point $M_{+}$, where $w$ evolves smoothly from 0 to 1 without ever passing by a dark energy phase. After the quantum bounce, the system emerges from $S_{-}$, and follows a dark energy phase until reaches the future dust attractor $M_{+}$.

Case B is the physically interesting solution. First because it can describe the present observed acceleration of the Universe, as long as a dark energy era takes place in the expanding phase. Second because there is no dark energy behavior of the scalar field in the contracting phase, avoiding the problem concerning the quantum vacuum prescription for the initial quantum state of cosmological perturbations when dark energy is present. There are other proposals of bouncing models with dark energy phase [92-94], motivated by different physical effects. The interesting aspect of the present model is that, with a single canonical scalar field with a simple potential, it was possible to model dark energy at large scales in the expanding phase of a bouncing model, and a dust fluid in the asymptotic past of the contracting phase of the same model.

Figure 8 shows an example of an exact Bohmian trajectory. Note that they satisfy almost everywhere the classical constraint $x^{2}+y^{2}=1$, except near the singularity, where the quantum bounce takes place, and the model goes from the region $x \approx 1$ to the region where $x \approx-1$. Hence, the $\mathrm{dBB}$ quantum approach to this classical model opened a new possibility for bouncing scenarios with dark energy: the dark energy behavior may be a feature only of the expanding phase, where it can model the present observed accelerated expansion, and it was absent in the contracting phase. In the far past of the contracting phase, the scalar field behaves like dust (running back in time, the dust repeller becomes an attractor), and vacuum initial conditions can be easily imposed in this era. Consequently, we get a well-posed problem to calculate the observed spectrum and amplitude of scalar cosmological perturbations in bouncing models with dark energy. 


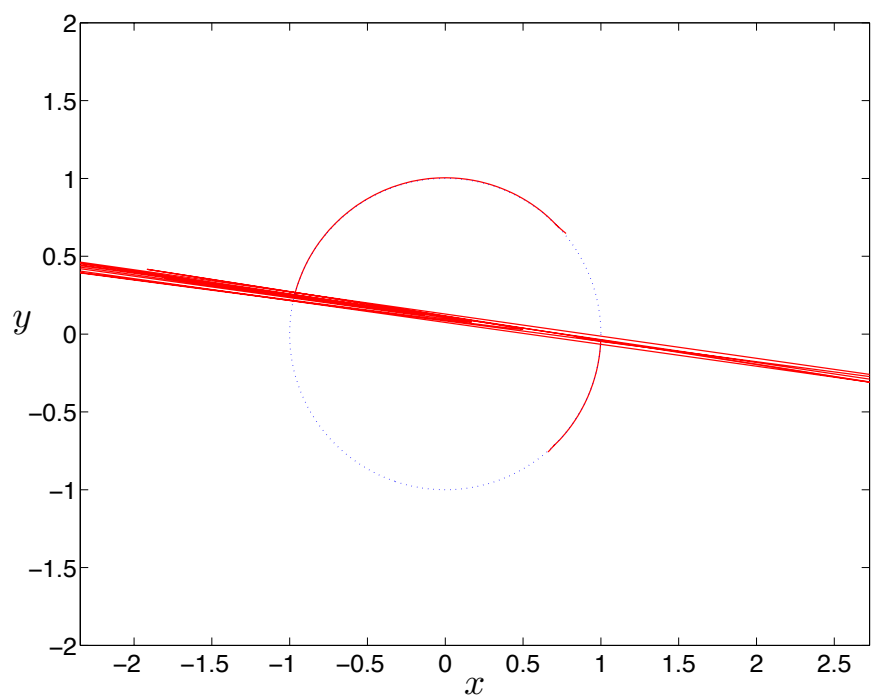

Figure 8. Bohmian trajectory for an exact solution [91]. It starts in the asymptotic past in the neighborhood of the critical point $(1 / \sqrt{2},-1 / \sqrt{2})$, and it ends in the asymptotic future in the neighborhood of the critical point $(1 / \sqrt{2}, 1 / \sqrt{2})$. Classical dynamics is valid almost everywhere, except near the singularity, where quantum effects become important and a bounce takes place. Note that the classical constraint $x^{2}+y^{2}=1$ ceases to be satisfied in the quantum region.

Let us now calculate the amplitudes of scalar perturbations and primordial gravitational waves in this model. We mentioned in Section 3 that classical bounces with a canonical scalar field normally presents excess of gravitational waves, the ratio $r=T / S$ is of order 1 , contrary to observations, where $T, S$ are the amplitudes of primordial gravitational waves and scalar perturbations, respectively. We will see that in a quantum bounce this is not the case [89].

The primordial gravitational waves are described by the variable $\mu$, which satisfies similar equations as the Mukhanov-Sasaki variable $v$, with the scale factor $a$ playing the role of $z$, see Section 3:

$$
\begin{aligned}
& v_{\mathbf{k}}^{\prime \prime}+\left(k^{2}-\frac{z^{\prime \prime}}{z}\right) z_{\mathbf{k}}=0, \\
& \mu_{\mathbf{k}}^{\prime \prime}+\left(k^{2}-\frac{a^{\prime \prime}}{a}\right) \mu_{\mathbf{k}}=0 .
\end{aligned}
$$

The approach to the quantization of $v$ can be used for $\mu$, hence the initial condition of an adiabatic vacuum state is also imposed for the tensor modes.

In order to understand qualitatively the final results, let us discuss what happens near the quantum bounce. When we get closer to the bounce in the contracting phase, the curvature of space-time increases a lot, and one is in the regime where $z^{\prime \prime} / z \gg k^{2}$ and $a^{\prime \prime} / a \gg k^{2}$, the super-Hubble behavior. As we have seen in Section 3, the solutions for the scalar and tensor perturbations at leading order in this regime are, respectively,

$$
\begin{aligned}
\zeta_{k} & \equiv \frac{v_{k}}{z} \approx A_{k}^{(1)}+A_{k}^{(2)} \frac{1}{R_{H}} \int \frac{\mathrm{d} t}{x^{2} a^{3}}, \\
h_{k} & \equiv \frac{\mu_{k}}{a} \approx B_{k}^{(1)}+B_{k}^{(2)} \frac{1}{R_{H}} \int \frac{\mathrm{d} t}{a^{3}},
\end{aligned}
$$

where $x$ was defined in Equation (112).

In the classical contracting branch of case B, $x$ varies between $1 / \sqrt{2}$ and 1 , while the scale factor goes through a large contraction. The value of this integral will be dominated by the values of $a$ near the bounce phase, where $a$ attains its smallest value. One can see from Equation (119) that what differentiates the evolution of $\zeta_{k}$ and $h_{k}$ in this region is the 
presence of $x$ in the expression for $\zeta$. As $x \approx 1$ classically, they will be very close during the whole evolution if the bounce is classical as well. Hence one gets the ratio $r=T / S \approx 1$, contrary to the observation limit $r<0.1$. This is the origin of the problem with classical bounce with canonical scalar fields. However, in a quantum bounce, when the quantum phase begins, the value of $x$ is no longer restricted to $(1 / \sqrt{2}, 1)$, see Figure 8 . In fact, it can be very small, and increase the scalar perturbation amplitudes relative to the tensor perturbation amplitudes. Indeed, this was calculated numerically, and the results can be seen in Figure 9. The curve shows that the presence of $1 / x^{2}$ in the aforementioned integral will result in a sharp increase in the scalar perturbation amplitude around $\left|\alpha-\alpha_{b}\right| \approx 10^{-1}$, where $\alpha_{b}$ is the value of the scale factor at the bounce. This effect takes place close to the bounce.

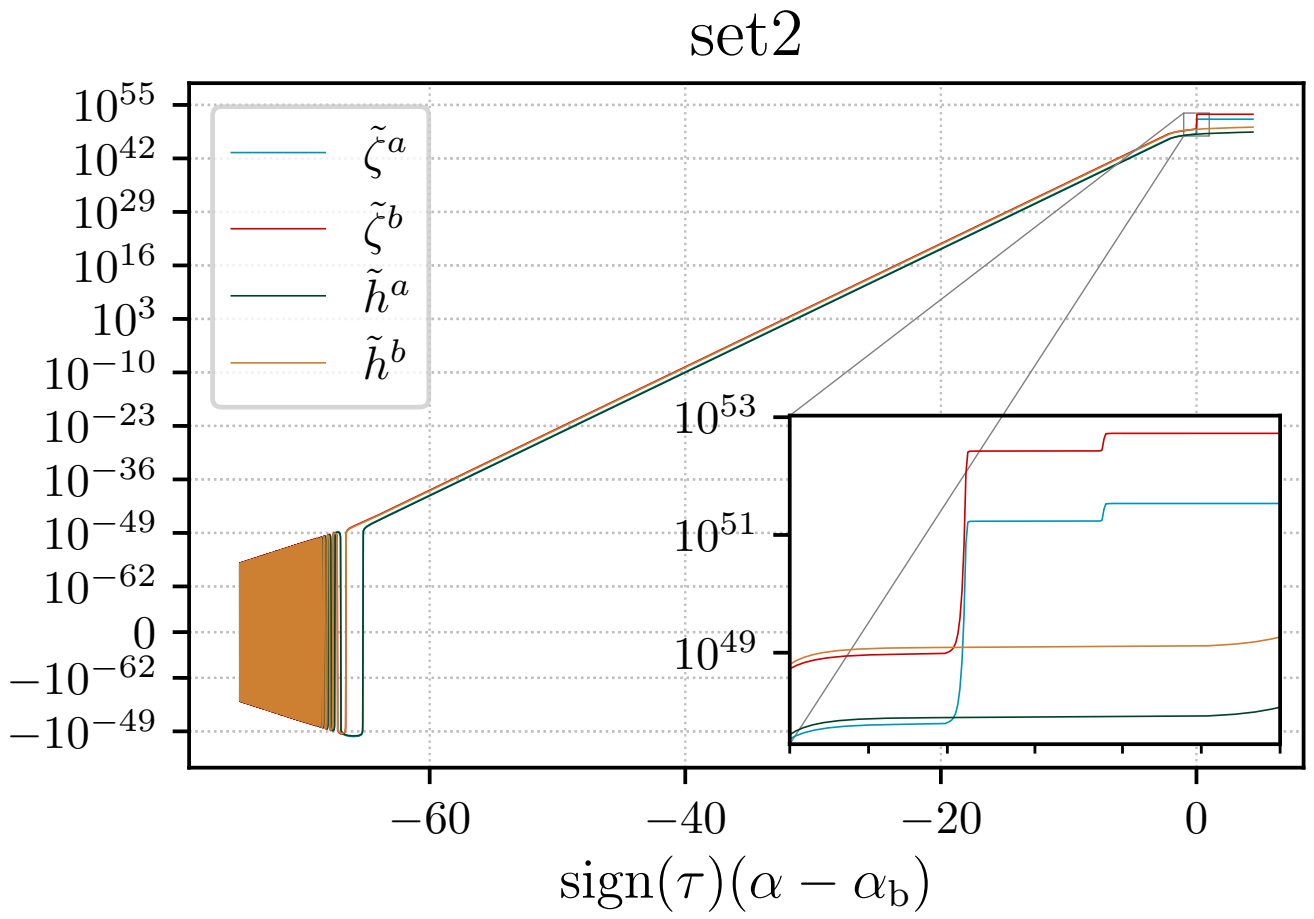

Figure 9. Evolution of scalar and tensor perturbations in the background of case B. Scalar and tensor perturbations grow almost at the same rate during classical contraction, but at the quantum bounce the scalar perturbations are enormously enhanced over the tensor perturbations due to the quantum effects (shown in the detail of the figure). After the bounce, the perturbations get frozen. The final amplitudes of both perturbations are compatible with observations. The indices $a$ and $b$ refer to the real and imaginary parts of the perturbation amplitudes.

This is a very important result. Firstly, it shows that quantum cosmological effects may solve problems which plague classical bouncing models, namely, large ratios of tensor to scalar perturbations amplitudes. More than this, it shows that features of quantum Bohmian trajectories can lead to observational consequences, and explain involved cosmological issues. Whether these conclusions can also be reached under other quantum or classical frameworks, is something yet to be verified. Nevertheless, the present model is a concrete example on how a quantum cosmological effect can be amplified to yield observable consequences.

The free parameters of the theory can be adjusted to yield the right amplitudes and spectral indices of scalar and tensor perturbations. In order to obtain the spectral index $n_{s}$ compatible with Planck observations, $n_{s}=0.9652 \pm 0.0042$, one must have $\lambda^{2}=2.9914 \pm 0.0010$. The curvature scale at the bounce must be of the order of $10^{3} l_{p}$ in order to yield the correct amplitude of scalar perturbations, $A_{s} \approx 2.1 \times 10^{-9}$, but the 
bounce itself must be steep in order to obtain sufficient amplification of scalar perturbations over tensor perturbations, as presented in Figure 9. See Reference [89] for details. Hence, with a single scalar field with a simple potential analyzed in the dBB framework, it was possible to construct a sensible bouncing model with dark energy behavior and correct and well defined perturbation amplitudes of quantum mechanical origin.

\section{Baryogenesis and Magnetogenesis}

As the curvature scale around the bounce is very high, some new physical effects may occur, like quantum particle creation [95,96]. In this section, I will describe processes like baryogenesis and magnetogenesis, which can be in accordance with present observations, and/or lead to new observational effects.

\subsection{Baryogenesis}

The baryon - anti-baryon asymmetry (excess of matter over antimatter in the Universe) is indicated by many observations, see References $[23,97,98]$, yielding the baryon-to-entropy ratio is $n_{B} / s=9.2_{-0.4}^{+0.6} \times 10^{-11}$. Its origin is still under debate, with no definite answer. The approaches are usually based on the Sakharov conditions [99] for the origin of this asymmetry. However, the investigations based on the Standard Model of Particle Physics (SMPP) do not yield sufficient baryon asymmetry, see Refs [100-102]. Hence, there has been investigations exploring physics beyond the SMPP, like in References [103-105].

In this subsection, I will present the results concerning baryogenesis in a bouncing background, assuming the so called gravitational baryogenesis [106], which also goes beyond SMPP by introducing a coupling between the derivative of the Ricci scalar and the baryon number current, and violates CPT symmetry in a dynamical universe. This interaction induces changes in the thermal equilibrium distributions, resulting in a nonzero net baryon number. I would like to remark that a purely radiation dominated primordial universe cannot produce sufficiently baryon asymmetry, even within gravitational baryogenesis, but it can be effective in bouncing model, as it must necessarily goes beyond classical radiation domination evolution in order for the very existence of the bounce, see Reference [107] in the context of Loop Quantum Cosmology.

I will assume the coupling term to be a CP-violating interaction with the form, as proposed in Reference [106],

$$
\frac{1}{M_{*}^{2}} \int d^{4} x \sqrt{-g}\left(\partial_{\mu} R\right) J^{\mu},
$$

where $M_{*}$ is the cutoff energy scale of the effective theory. This term, in an expanding universe, also dynamically breaks CPT and favors a net asymmetry towards the production of baryons over anti-baryons. This happens because the $J^{0}\left(=n_{B}\right)$ term has a different sign for matter versus antimatter, and as it will be demonstrated here, it can be used to calculate the net asymmetry of matter and antimatter once the universe reaches a decoupling temperature for this effective theory. Then, if we also assume that the characteristic timescale $\tau$ of the interaction runs faster than the expansion rate of the universe, that is,

$$
\tau<<H^{-1},
$$

where $H$ is the Hubble constant, we can use thermal equilibrium relations to calculate the baryon-to-entropy ratio. Identifying the term multiplying the baryon density $n_{B}$ as its chemical potential $\mu_{B}$, namely

$$
\mu_{B}= \pm \frac{\dot{R}}{M_{*}^{2}},
$$

where the plus (minus) sign stands for particles (anti-particles), the baryon-to-entropy ratio at decoupling temperature reads 


$$
\frac{n_{B}}{s}=\frac{15 g_{B} \mu_{B}}{4 \pi^{2} g_{*} T}=\left.\frac{15 g_{B}}{4 \pi^{2} g_{*}} \frac{\dot{R}}{M_{*}^{2} T}\right|_{T=T_{D}}{ }^{\prime}
$$

where $s$ is the entropy, $g_{*}$ and and $g_{B}$ are the number of degrees of freedom of all species and baryons in the hot Universe, respectively, and $T_{D}$ is the effective interaction decoupling temperature. Once the temperature drops below $T_{D}$, the effective baryon production freezes, and the resulting asymmetry is then preserved. In order to look for physical solutions, we must require this ratio to be the observed $n_{B} / s \approx 9 \times 10^{-11}$, and then look to the parameter space in order to obtain the region satisfying it.

I will work with $g_{*} \approx 100$, and $g_{b} \approx 1$. Equation (124) depends on the physical variables $x_{b}, T_{D}$ and $M_{*}$. The former lies in the range $10^{15} \leq x_{b} \leq 10^{31}$, where the lower limit restricts the calculation to well before the start of nucleosynthesis, whereas the upper limit is due to the Planck scale. The decoupling temperature $T_{D}$ lies in the range $10 \mathrm{TeV} \leq T_{D} \leq 10^{19} \mathrm{GeV}$, where the lower limit avoids observable effects in LHC, and the upper limit is the Planck scale. Finally, for the cutoff energy scale $M_{*}$ we set the range $10^{-16} M_{p} \leq M_{*} \leq M_{p}$, for the same reasons as $T_{D}$. Therefore, we have

$$
\begin{aligned}
10^{15} \leq x_{b} & \leq 10^{31}, \\
10^{7} \leq \bar{T}_{D} & \leq 10^{22}, \\
10^{-16} \leq \bar{M}_{*} & \leq 1,
\end{aligned}
$$

where $\bar{T}_{D}=T_{D} / \mathrm{MeV}$ and $\bar{M}_{*}=M_{*} / M_{\mathrm{p}}$ are dimensionless quantities.

I will consider the radiation dominated quantum bounce $(\omega=1 / 3)$ which, in conformal dimensionless time, reads:

$$
a(\bar{\eta})=a_{b} \sqrt{1+\bar{\eta}^{2}}
$$

where $\bar{\eta}=\eta / \eta_{b}$. Far from the bounce, we can relate the cosmic time $t$ with $\bar{\eta}$ as:

$$
t(\bar{\eta})=\frac{a_{b} \eta_{b} \bar{\eta}^{2}}{2}
$$

where $\eta_{b}$ can be related to the Hubble radius $R_{H_{0}}=1 / H_{0}$ and the radiation density today $\Omega_{r 0}$ as

$$
\eta_{b}=\frac{R_{H_{0}}}{a_{b} x_{b}^{2} \sqrt{\Omega_{r 0}}}
$$

see Reference [108] for details. Considering the values $H_{0}=1.22 \times 10^{-61} M_{p}, M_{p}=$ $1.22 \times 10^{22} \mathrm{MeV}$, from Equation (129) one obtains

$$
\bar{\eta}(\bar{T})=\frac{1.0 \times 10^{-10} x_{b}}{\bar{T}} .
$$

For the the symmetric scale factor given by Equation (128), using Equation (131) in Equation (124), the result for $n_{B} / s$ far from the bounce $(\bar{\eta} \gg 1)$ reads:

$$
\frac{n_{B}}{s}=6.2 \times 10^{-86} \frac{\bar{T}_{D}^{7}}{x_{b}^{2} \bar{M}_{*}^{2}},
$$

where we used $\Omega_{r 0}=8 \times 10^{-5}$. Finally, $n_{B} / s$ is given in terms of the parameters $x_{b}, \bar{T}_{D}$ and $\bar{M}_{*}$.

One must notice that Equation (131) was obtained through an expansion assuming that $\bar{\eta} \gg 1$, which imposes the condition (To apply the condition $\bar{\eta} \gg 1$, we assumed $\bar{\eta}>10$ as sufficient because we expand terms $\left.1+\bar{\eta}^{2}\right):$

$$
\frac{x_{b}}{\bar{T}} \gtrsim 1.0 \times 10^{11} .
$$


Hence, this inequality must be considered together with conditions (125)-(127) when we look for the regions of interest in the parameter space.

In Figure 10, we present a region plot for $\bar{T}_{D} \times x_{b}$ and lines for constant values of $\bar{M}_{*}$. The region of parameters that give $n_{B} / s \approx 9 * 10^{-11}$ are the values of the gray region that are crossed by the constant $\bar{M}_{*}$ lines.

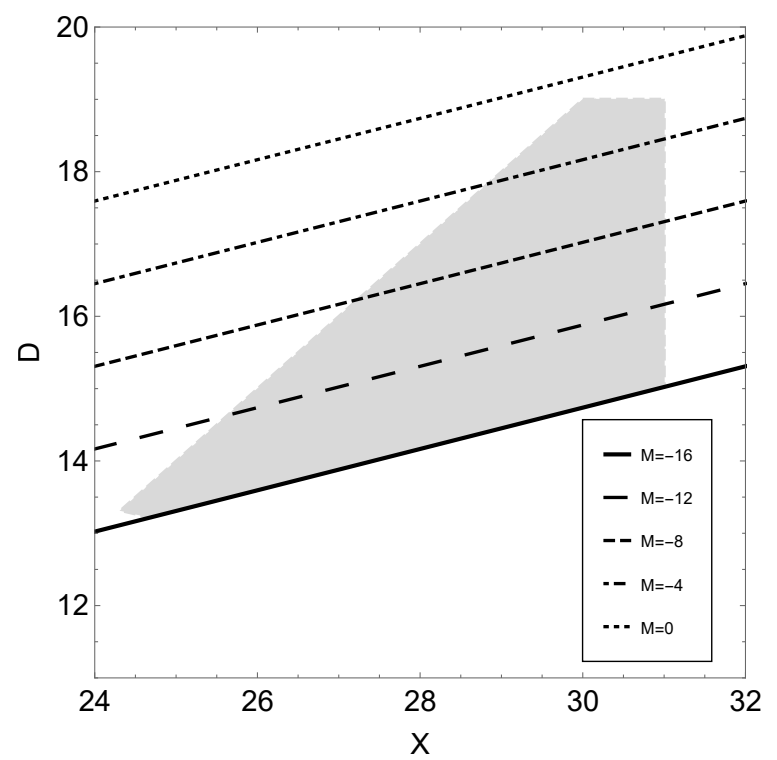

Figure 10. Parameter space of $x_{B}, T_{D}, M_{*}$ that that gives $n_{B} / s \approx 9 * 10^{-11}$. They are parameterized by $X=\log \left(x_{b}\right), D=\log \left(\bar{T}_{D}\right)$ and $M=\log \left(\bar{M}_{*}\right)$, respectively.

One can see that there is a large range of allowed values for the free parameters, with a preference for high energy bounces and small cut-off scales. The details of this analysis can be found in Reference [108], with extensions to other quantum bouncing models, and other baryon couplings.

\subsection{Magnetogenesis}

One of the main issues concerning contemporary cosmology consists the origin of magnetic fields, which are observed in a variety of scales in the Universe. In particular, there are several observations consistent with weak $\sim 10^{-16}$ Gauss fields in the intergalactic medium, coherent on Mpc scales. Since such fields remained largely undisturbed during the cosmological evolution (as opposed to those in the presence of structure), they offer a window to their origin, which is generally assumed to be primordial. Primordial seed fields, which may be amplified later by the dynamo mechanism, are generated before structure formation. For reviews, see References [109-111]. However, since minimally-coupled electromagnetism is conformally invariant, the expansion cannot affect its vacuum state. Hence such invariance must be broken in order to generate seed magnetic fields.

In this subsection I will study magnetic field production when conformal invariance is broken through a non-minimal coupling with gravity. This possibility has been widely studied in the case of inflationary models (see References [112-114], among others). However, inflationary magnetogenesis is not free of problems. Among these, we can mention an exponential sensitivity of the amplitude of the generated magnetic field with the parameters of the inflationary model [115], the strong coupling problem [116], and the limits in the magnetic field strength coming from the gravitational backreaction of the electric fields that are produced simultaneously with the magnetic fields [117]. Hence, instead of an inflationary model, a bouncing universe in conjunction with a coupling of the type $R F_{\mu v} F^{\mu v}$ will be used here to study the production of seed magnetic fields. This coupling, theoretically motivated by vacuum polarization in quantum electrodynamics (QED) in a curved background [118], introduces a mass scale to be fixed by observations. 
As the bounce background, I will take the quantum bounce with a pressureless perfect fluid (necessary to yield almost scale invariant spectrum of scalar perturbations), without any radiation fluid, as long as the electromagnetic field will be generated along the evolution of the model. It will be useful to express the scale factor as $a(t) \equiv a_{0} Y(t)$, with

$$
Y(t)=\frac{1}{x_{b}}\left(1+\frac{t^{2}}{t_{b}^{2}}\right)^{1 / 3},
$$

where $x \equiv a_{0} / a, t$ is cosmic time, and $t_{b} \equiv 2 L_{b}$, with $L_{b}$ the curvature scale at the bounce $\left(L_{b} \equiv 1 / \sqrt{|R(0)|}\right.$ where $R$ is the four-dimensional Ricci scalar) satisfying $10^{3} t_{\text {Planck }}<t_{b}<$ $10^{40} t_{\text {Planck, }}$, the limits related, as usual, to the Planck and nucleosynthesis era.

For the subsequent calculations, it is convenient to define parameters that are directly related to observations. Let us first write down the Friedmann equation as

$$
H^{2}=H_{0}^{2} \Omega_{m} x^{3},
$$

with $\Omega_{m}$ the dimensionless dark matter density today. Then, from the expansion of $a(t)$ for large values of $t$, it follows that

$$
H^{2} \approx \frac{4}{9 t_{b}^{2}}\left(\frac{x}{x_{b}}\right)^{3}
$$

Now, using $H_{0}=70 \mathrm{~km} \mathrm{~s}^{-1} \mathrm{Mpc}^{-1}$ and the lower bound on $t_{b}$, it is straightforward to derive an upper limit on $x_{b}$ by equating Equations (135) and (136),

$$
\Omega_{m}=\frac{4}{9} \frac{1}{t_{b}^{2} x_{b}^{3} H_{0}^{2}} \Longrightarrow x_{b}<\frac{10^{38}}{\Omega_{m}^{1 / 3}} .
$$

For later convenience, we define $R_{H_{0}} \equiv H_{0}^{-1}, t_{s} \equiv t / R_{H_{0}}$, and $\alpha \equiv R_{H_{0}} / t_{b}$, and rewrite $Y(t)$ as

$$
Y\left(t_{s}\right)=\frac{1}{x_{b}}\left(1+\alpha^{2} t_{s}^{2}\right)^{1 / 3}
$$

with

$$
\alpha=\frac{3}{2} \sqrt{\Omega_{m} x_{b}^{3}} .
$$

I will take the non-minimal coupling Lagrangian

$$
\mathcal{L}=-f F_{\mu v} F^{\mu v},
$$

where

$$
f \equiv \frac{1}{4}+\frac{R}{m_{\star}^{2}},
$$

and $m_{\star}$ is a mass scale to be determined by observations. The non-minimal coupling breaks conformal invariance, and paves the way to the production of primordial electromagnetic fields.

The equations of motion for the electromagnetic field that follow from Equation (140) are

$$
\partial_{\mu}\left(\sqrt{-g} f F^{\mu \nu}\right)=0,
$$


where the field $F_{\mu \nu}$ is expressed in terms of the gauge potential $A_{\mu}$ as $F_{\mu \nu}=\partial_{\mu} A_{\nu}-\partial_{\nu} A_{\mu}$. To quantize the electromagnetic field, we expand the operator associated to the spatial part of the vector potential as

$$
\hat{A}_{i}(t, \mathbf{x})=\sum_{\sigma=1,2} \int \frac{d^{3} k}{(2 \pi)^{3 / 2}}\left[\epsilon_{i, \sigma}(\mathbf{k}) \hat{a}_{\mathbf{k}, \sigma} A_{k, \sigma}(t) e^{i \mathbf{k} \cdot \mathbf{x}}+\text { H.C. }\right],
$$

where $\epsilon_{i, \sigma}(\mathbf{k})$ are two orthonormal and transverse vectors which are constant across spatial sheets (they have zero Lie derivative with respect to the spatial foliation vector field) and H.C. stands for the Hermitian conjugate. The operators $\hat{a}_{\mathbf{k}, \sigma}$ and $\hat{a}_{\mathbf{k}, \sigma}^{+}$are respectively the annihilation and creation operators. They satisfy $\left[\hat{a}_{\mathbf{k}, \sigma}, \hat{a}_{\mathbf{k}^{\prime}, \sigma^{\prime}}^{+}\right]=\delta_{\sigma \sigma^{\prime}} \delta\left(\mathbf{k}-\mathbf{k}^{\prime}\right),\left[\hat{a}_{\mathbf{k}, \sigma}, \hat{a}_{\mathbf{k}^{\prime}, \sigma^{\prime}}\right]=$ 0 , and $\left[\hat{a}_{\mathbf{k}, \sigma^{\prime}}^{+} \hat{a}_{\mathbf{k}^{\prime}, \sigma^{\prime}}^{\dagger}\right]=0$. Note that in the equations above we adopted the Coulomb gauge with respect to the cosmic time foliation $\left(A_{0}=0\right.$ and $\left.\partial_{i} A^{i}=0\right)$. The time-dependent coefficients $A_{k, \sigma}(t)$ and their associated momenta $\Pi_{k, \sigma} \equiv 4 a f A_{k, \sigma}^{\prime}(t)$ must satisfy

$$
A_{k, \sigma}(t) \Pi_{k, \sigma}^{*}(t)-A_{k, \sigma}^{*}(t) \Pi_{k, \sigma}(t)=i,
$$

for each $k$ and $\sigma$. It should be emphasized that the quantization of the gauge-fixed electromagnetic field in the absence of charges is equivalent to that of two free real scalar fields. Consequently, the choice of vacuum for each polarization $\sigma$ corresponds to the choice of vacuum of each scalar degree of freedom. However, using the fact that we are dealing with an isotropic background, there is no reason to make different choices of vacuum for different polarizations. For this reason, we choose a single time-dependent coefficient to describe both polarizations, that is, $A_{k, 1}=A_{k, 2} \equiv A_{k}$. Therefore, the same vacuum is chosen for both polarizations. Now, inserting this decomposition in Equation (142), we get the equation governing the evolution of the modes $A_{k}(t)$

$$
\ddot{A}_{k}+\left(\frac{\dot{a}}{a}+\frac{\dot{f}}{f}\right) \dot{A}_{k}+\frac{k^{2}}{a^{2}} A_{k}=0 .
$$

Defining

$$
k_{s} \equiv k R_{H}, \quad A_{s k}\left(t_{s}\right) \equiv \frac{A_{k}\left(t_{s}\right)}{\sqrt{x_{b} R_{H_{0}}}},
$$

where $R_{H}=R_{H_{0}} / a_{0}$ is the co-moving Hubble radius today, the differential Equation (145) can be written as

$$
A_{s k}^{\prime \prime}+\left(\frac{Y^{\prime}}{Y}+\frac{f^{\prime}}{f}\right) A_{s k}^{\prime}+\frac{k_{s}^{2}}{Y^{2}} A_{s k}=0,
$$

where a prime denotes the derivative with respect to $t_{s}$. The coupling (141) then takes the form

$$
f=\frac{1}{4}\left[1+C^{2} \frac{\alpha^{2} t_{s}^{2}+3}{\left(\alpha^{2} t_{s}^{2}+1\right)^{2}}\right] ; \quad \text { with } C^{2} \equiv \frac{4}{3} \frac{\ell_{*}^{2}}{t_{b}^{2}}, \quad \ell_{*} \equiv \frac{1}{m_{*}}
$$

An upper limit on $C$ can be straightforwardly derived from Equation (148). Since any contribution to the usual Maxwell's equations at BBN must be negligible, we impose the second term in Equation (148) to be smaller than $10^{-2}$ at BBN. Together with the fact that $\alpha^{2} t_{s}^{2} \gg 1$ at this time, we get

$$
C<10^{-19} x_{b}^{3 / 2}
$$


The energy densities of the electric and magnetic fields are respectively given by

$$
\begin{aligned}
& \rho_{E}=\frac{f}{8 \pi} g^{i j} A_{i}^{\prime} A_{j}^{\prime}, \\
& \rho_{B}=\frac{f}{16 \pi} g^{i j} g^{l m}\left(\partial_{j} A_{m}-\partial_{m} A_{j}\right)\left(\partial_{i} A_{l}-\partial_{l} A_{i}\right),
\end{aligned}
$$

where $g^{i j}=\delta^{i j} / a^{2}$ are the spatial components of the inverse metric. To find the spectral energy densities, we first insert expansion (143) into $\rho_{E}$ and $\rho_{B}$. The resulting operators $\hat{\rho}_{E}$ and $\hat{\rho}_{B}$ upon quantization are

$$
\begin{aligned}
& \hat{\rho}_{B}=\frac{f}{2 \pi^{2} R_{H_{0}}^{4} Y^{4}} \int \operatorname{dln} k\left|A_{s k}\right|^{2} k^{5}, \\
& \hat{\rho}_{E}=\frac{f}{2 \pi^{2} R_{H_{0}}^{4} Y^{2}} \int \operatorname{dln} k\left|A_{s k}^{\prime}\right|^{2} k^{3} .
\end{aligned}
$$

We now evaluate the expectation value of the two densities in vacuum, defined by $\hat{a}_{\mathbf{k}, \sigma} \mid 0>=0$, and define the spectra as

$$
\mathcal{P}_{i} \equiv \frac{\mathrm{d}<0\left|\hat{\rho}_{i}\right| 0>}{\mathrm{d} \ln k}, \quad i=E, B .
$$

This yields the magnetic and electric spectra, respectively

$$
\begin{aligned}
& \mathcal{P}_{\mathcal{B}} \equiv B_{\lambda}^{2}=\frac{f}{2 \pi^{2} R_{H_{0}}^{4}} \frac{\left|A_{s k}\right|^{2}}{Y^{4}} k^{5}, \\
& \mathcal{P}_{\mathcal{E}} \equiv E_{\lambda}^{2}=\frac{f}{2 \pi^{2} R_{H_{0}}^{4}} \frac{\left|A_{s k}^{\prime}\right|^{2}}{Y^{2}} k^{3}=\frac{1}{2 \pi^{2} R_{H_{0}}^{4}} \frac{\left|\Pi_{s k}\right|^{2}}{f Y^{4}} k^{3} .
\end{aligned}
$$

In the last line, we also expressed $\mathcal{P}_{\mathcal{E}}$ in terms of the momentum canonically conjugate to the gauge field $\Pi_{s k}=Y f A_{s k}^{\prime}$ (see Reference [119]), which is nothing but the electric field mode itself.

Finally, we can express the magnetic and electric fields, $B_{\lambda}$ and $E_{\lambda}$, using $H_{0}^{2}=$ $1.15 \times 10^{-64} \mathrm{G}$

$$
\begin{aligned}
& B_{\lambda}=\sqrt{\frac{f}{2 \pi^{2}}} \frac{\left|A_{s k}\right|}{Y^{2}} k^{5 / 2} 1.15 \times 10^{-64} \mathrm{G}, \\
& E_{\lambda}=\sqrt{\frac{1}{2 \pi^{2} f}} \frac{\left|\Pi_{s k}\right|}{Y^{2}} k^{3 / 2} 1.15 \times 10^{-64} \mathrm{G} .
\end{aligned}
$$

Nonsingular models are likely to ease both the problem of the exponential sensitivity of the result and the strong coupling problem, since they expand slower than inflationary models [120-122]. In order to verify all that, we numerically evolved the equations. The main results are displayed in the figures below. For details, see Reference [119]. It was also verified that backreaction is not an issue for the model chosen here.

At the beginning of the evolution, modes are not excited. Only vacuum fluctuations are present, with the usual $k^{4}$ spectrum, increasing as $Y^{-4}$ due to contraction. When the coupling $f$ becomes relevant, the magnetic field power spectrum begins to increase faster, since $f$ is a growing function in the contracting phase, while the electric field power spectrum presents a slower increment, up to the time when $\Pi_{k}$ also begins to increase. After the bounce the situation is reversed, because $f$ is a decaying function of time in the expanding phase: the electric power spectrum decreases much slower than the magnetic one. It can be seen that the decay is mild, going as $t^{-2 / 3}$, when $1 / \alpha<t<C / \alpha$, opening a window in time where the electric spectrum has a significantly higher contribution than the magnetic one, see Figure 11. It was verified that for larger $C^{\prime}$ s, the decrease of the electric 
contribution at late times happens later, and the total electromagnetic power spectrum is more important. Higher values of $x_{b}$ imply an overall stronger total electromagnetic power spectrum, but with a stronger decrease rate at late times.

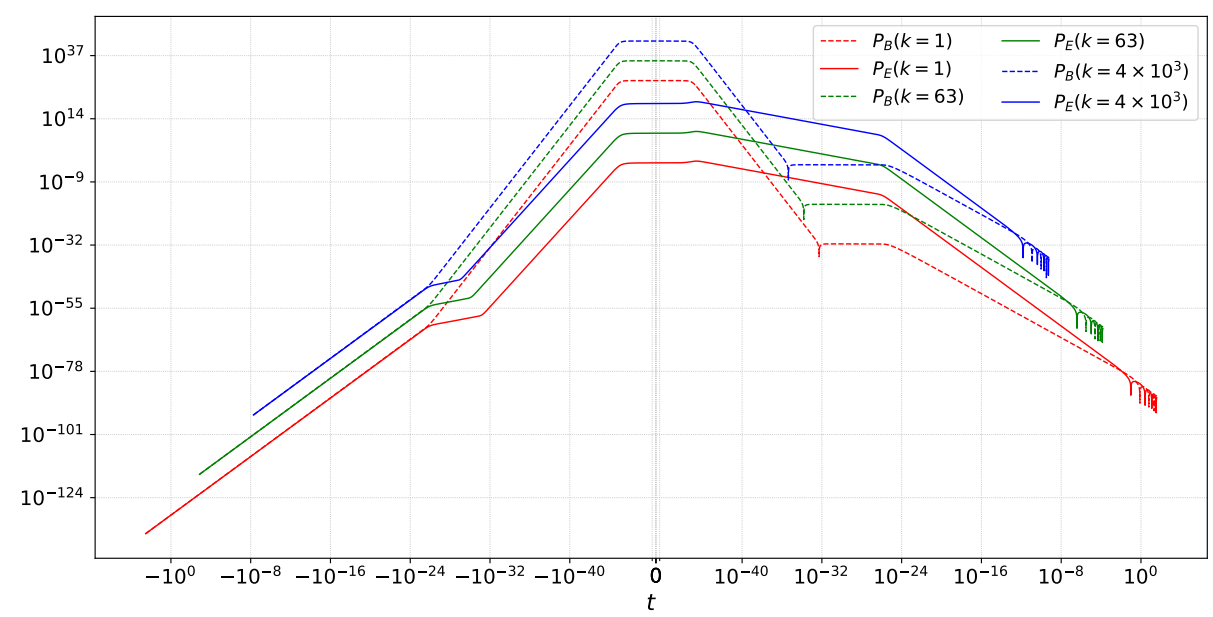

Figure 11. Evolution of the magnetic (dashed lines) and electric (continuous line) power spectra for $C=10^{19}$ and $x_{b}=10^{30}$.

Another interesting aspect of the magnetic and electric power spectra is their dependence in terms of $k$, shown in Figure 12. As predicted by analytical estimates, see Reference [119], we obtain the spectral index $n_{B}=6$, see Figure 12. This is typical of non-helicoidal and causally generated magnetic fields, as noted in Reference [123].

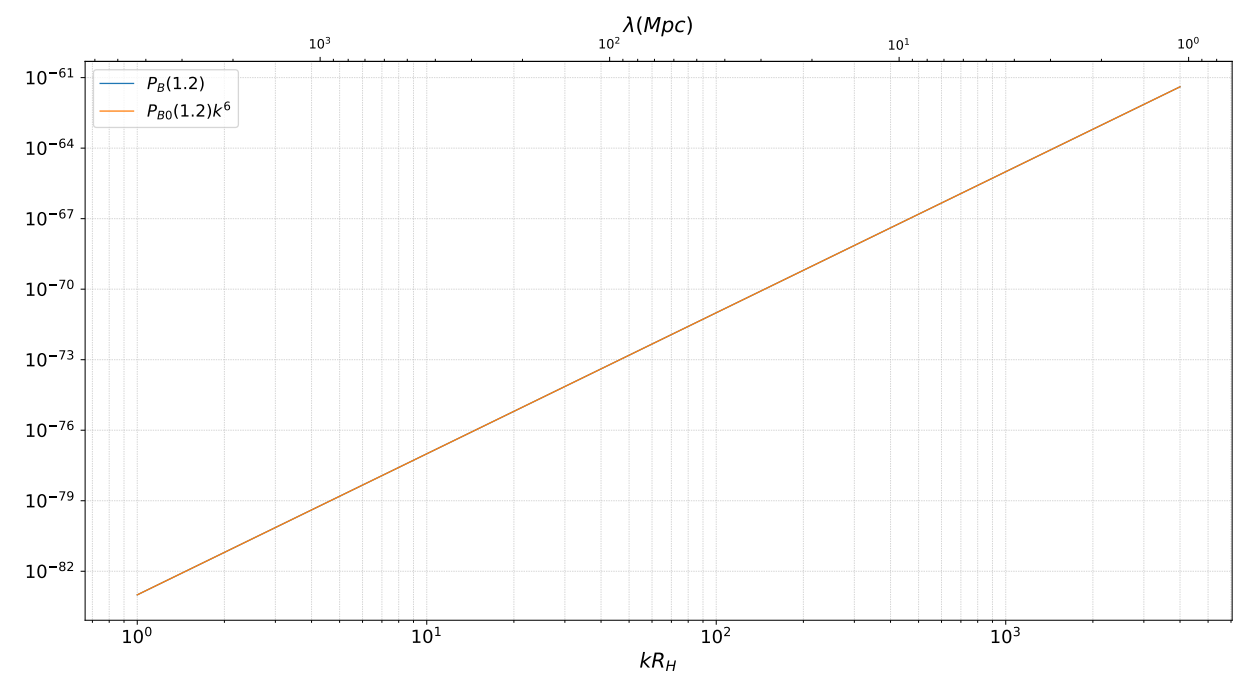

Figure 12. Behavior of the magnetic power spectrum today from (155) (blue) for $C=10^{23}$ and $x_{b}=10^{30}$. It is perfectly compatible with a power-law with spectral index $n_{B}=6$.

The theoretical and observational limits are used in Figure 13 in order to constrain the region in parameter space for which consistent values of magnetic seed fields, evaluated today, are obtained at $1 \mathrm{Mpc}$. The upper value $x_{b} \lesssim 10^{38}$ comes from Equation (137) reflecting the earliest possible time for the bounce to occur. It is denoted "Planck Scale" in the graph. There is another limit set to preserve nucleosynthesis denoted "BBN". This can be derived by plugging Equation (149) into Equation (159) presented below, giving $m_{\star}=10^{-19} m_{e}$. 
In order to infer the allowed mass scales for the minimal coupling, one can use, for instance, the relation between $C$ and $m_{\star}$ coming from Equation (148) to show that

$$
\frac{m_{\star}}{m_{e}}=\frac{\alpha}{10^{38} C^{\prime}}
$$

where $m_{e}$ is the electron mass. The maximum mass allowed in this model is then $0.1 m_{e}$. Therefore, the value of the electron mass for $m_{\star}$ is not allowed by our model, a feature shared with power-law inflationary models $[112,113]$.

\section{$\log x_{b}$}

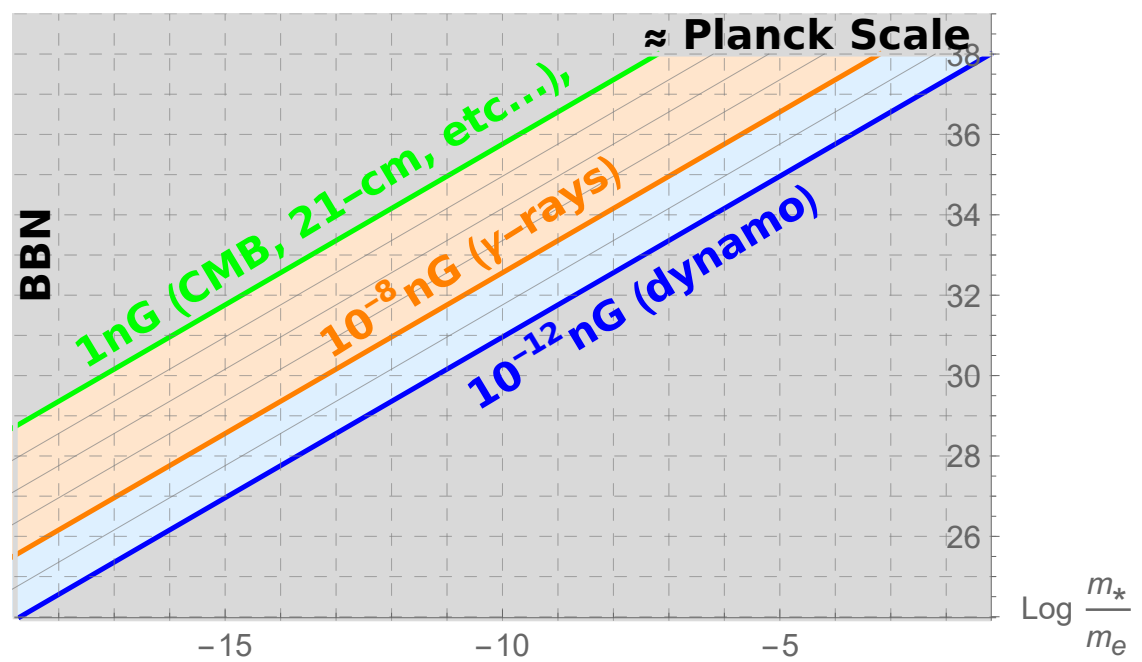

Figure 13. Parameter space with magnetic field amplitudes consistent with current limits at $1 \mathrm{Mpc}$. The blue region shows the allowed values to initiate the dynamo effect. The blue line is its theoretical lower limit [115]. The orange region represents allowed values coming from observations at large scales in voids, the orange line is the lower limit derived by blazars observations [124], and the green line is the upper limit derived using Ultra-High-Energy Cosmic Rays, Ultra-Faint Dwarf galaxies, 21-cm hydrogen lines, and so forth [125]. The orange and blue regions are overlapping. The grey shaded region denotes excluded values of the magnetic field. Each oblique grey line gives an amplitude for the magnetic field a hundred times higher than the lower line.

In order to avoid backreaction, new constraints on the parameters may arise. However, this is not the case with this model. Choosing the galactic scale (tens of kiloparsecs), $k \approx 10^{5}$, as the cut-off scale $k_{f}$ where these calculations can be trusted, yields the constraints:

(1) Backreaction of the magnetic field: $C^{2} x_{b}<10^{98}$. The values given in Figure 13 respect this constraint.

(2) Backreaction of the electric field: $C<10^{26.3}$, which is allowed in Figure 13.

In conclusion, there is no electromagnetic backreaction at the bounce. Also, the model is not exponentially dependent on the parameters, neither suffers from the strong coupling problem.

\section{Discussion and Conclusions}

This paper presented results and properties of a class of quantum bouncing models. They are obtained using a simple quantization procedure, with the compromise that the resulting models never get to close to Planck energy, where other more involved quantization schemes should have to be used: Dirac canonical quantization of a midisuperspace model composed of a inhomogeneous and isotropic background, together with small homogeneous perturbations. The results were obtained in the framework of the de Broglie-Bohm quantum theory, turning out to enormously simplify the calculations, conceptually and technically. 
Assuming a homogeneous and isotropic background with small inhomogeneous perturbations, it has been shown that the bouncing models studied are stable. Of course, if one relaxes isotropy, the Belinskii- Khalatnikov-Lifshitz (BKL) instability [126] may appear, and an ekpyrotic phase would have to be evoked [15]. However, as mentioned in the Introduction, any cosmological model has to face the issue concerning the large degree of homogeneity of the hot universe, which is infinitely more serious than the BKL problem. In fact, in order to get homogeneity, one has to turn identical the infinite possible functions of time per space point characterizing a general inhomogeneous geometry and matter fields. If there exists a physical mechanism or theory of initial conditions capable of justifying such an extreme fine-tuning of infinitely many time functions, then it would be natural to suppose that it could also justify the fine-tuning of the three remaining time functions characterizing the three directions of space. All cosmological models assume, without a really sound and established justification, as discussed in the Introduction, the initial homogeneity of initial conditions. If one further assumes isotropy, then it can be shown, for the models we are considering here, that the shear perturbation will never overcome the background degrees of freedom, even growing as fast as $a^{-6}$ in the contracting phase, because it is multiplied by the factor $\left(l_{p} / R_{H_{0}}\right)^{2}$, where $l_{p}$ is the Planck length and $R_{H_{0}}$ is the Hubble radius today, which is a very small number. In Reference [86] it was shown that for bounce scales not so close to the Planck length, which is the case of the models I have presented in this paper, the shear will always remain sufficiently small. It seems to me that adding an extra ekpyrotic scalar field, with suitable but ad hoc potential seems excessive to solve an issue (isotropy) which is far from being the most serious one, unless this extra field can also solve, or significantly ameliorate, the homogeneity problem, which is now under investigation [15]. It may also happen that some quantum gravity argument justifies the isotropy initial condition, without any new ad hoc ekpyrotic field.

Concerning observations, and comparing with inflation, besides solving the singularity problem by construction, bouncing models do not have the horizon, flatness, and structure formation problems. If the fluid that dominates the contracting phase when scales of cosmological interest cross the sound Hubble radius is pressureless, then an almost scale invariant spectrum of scalar cosmological perturbations emerges in the expanding phase. As in inflation, they emerge from quantum vacuum fluctuations, justifying an initial Gaussian distribution, and their amplitudes can be accommodated with CMBR observations with bounces in which their maximum energy density do not get very close to the Planck energy. This pressureless fluid can be dark matter, a component that we are already observing, without the need of evoking any new yet unobserved field, like the inflation (if one does not impose an ekpyrotic contracting phase). In this sense, bouncing models are conceptually simpler than inflation. However, the red-tilted spectrum of scalar perturbations was predicted as a natural result arising from any slow roll inflationary model [22]. In bouncing models, this is not the case. If the pressureless field is described by a scalar field with exponential potential, then one can adjust the exponential potential to fit the data, but, contrary to inflation, it is an ad hoc fixation, just to accommodate with observations. If it is a fluid, then the situation is yet more involved, as one would expect a blue-tilted spectrum, because one usually has, in this case, $w=p / \rho=c_{s}^{2}>0$. This could be overcome with some self-interaction of the fluid, or due to the presence of other components, like radiation or a cosmological constant, as discussed in Sections 3 and 5. Investigations about this issue must be deepened. A full comparison with all Planck angular power spectrum observations [23] in the fluid case with two components must be done, and it is work in progress. We have also seen that such quantum bouncing models are suitable backgrounds for baryogenesis and magnetogenesis to happen, once some proposed non-minimal coupling interactions are assumed.

Note that, in the fluid case, the quantum correction to the classical Friedmann equation works like a classical stiff matter fluid with negative energy $\rho_{q} \propto-1 / a^{6}$. This quantum effect was also found in other completely different quantum frameworks [127], as well as 
in loop quantum cosmology, if the background is dust (as it should be at large scales if the model leads to an almost scale invariant spectrum).

One very important line of research would be to find observables signing the existence of a contracting phase and/or a bounce in the history of the Universe. It seems that gravitational waves will not help, as bouncing models and inflation allow a variety of tensor perturbation configurations, unless, as we have seen, one finds a possibility of measuring primordial gravitational waves at frequencies above $1 \mathrm{~Hz}$, which is quite difficult, as they are stochastic. Other possibilities are non-gaussianities [128,129], and the role of dark energy in bouncing models. Concerning dark energy, we have seen that its presence in the contracting phase can indeed modify the spectrum of scalar perturbations at large scales, yielding oscillations, and, more important, it affects the very notion of an initial quantum vacuum state for the quantum perturbations, which becomes much more subtle. This can be a problem and/or an opportunity, as, in such a framework, dark energy is not only affecting the present and future of our universe, but it may also have played a fundamental role in the far past of our universe, as it can be deeply connected with the origin of structures in the Universe. Hence, if this is the case, detailed investigations about CMBR and its anisotropies can also give hints about the very nature of dark energy, which is note the case for inflation. I think a deep investigation of dark energy in bouncing models is one of the main promising lines of investigation in this area, not only conceptually, but also observationally.

Funding: This research received no external funding.

Acknowledgments: N.P.-N. acknowledges support of CNPq of Brazil under grant PQ-IB 309073/2017-0.

Conflicts of Interest: The author declares no conflicts of interest.

\section{References}

1. Ade, P.A.R.; Aghanim, N.; Arnaud, M.; Ashdown, M.; Aumont, J.; Baccigalupi, C.; Banday, A.J.; Barreiro, R.B.; Bartlett, J.G.; Bartolo, N.; et al. Planck Collaboration. Astron. Astrophys. 2016, A13, 594.

2. Cyburt, R.H.; Fields, B.D.; Olive, K.A.; Yeh, T. Big bang nucleosynthesis: Present status Rev. Mod. Phys. 2016, 88, 015004. [CrossRef]

3. DES Collaboration. Dark Energy Survey Year 1 Results: Cosmological constraints from cluster abundances and weak lensing. Phys. Rev. D 2020, 102, 023509. [CrossRef]

4. Abolfathi, B.; Aguado, D.S.; Aguilar, G.; Allende Prieto, C.; Almeida, A.; Ananna, T.T.; Anders, F.; Anderson, S.F.; Andrews, B.H.; Anguiano, B.; et al. The Fourteenth Data Release of the Sloan Digital Sky Survey: First Spectroscopic Data from the Extended Baryon Oscillation Spectroscopic Survey and from the Second Phase of the Apache Point Observatory Galactic Evolution Experiment. Astrophys. J. Suppl. Ser. 2018, 235, 19. [CrossRef]

5. Hollands, S.; Wald, R.M. Essay: An alternative to inflation. Gen. Rel. Grav. 2002, 34, 2043. [CrossRef]

6. Wald, R.M. The Arrow of Time and the Initial Conditions of the Universe. arXiv 2005, arXiv:gr-qc/0507094.

7. Fischer, A.E. Unfolding the singularities in superspace. Gen. Rel. Grav. 1983, 12, 1191. [CrossRef]

8. Green, M.B.; Schwarz, J.H.; Witten, E. Superstring Theory Vol. 1; Cambridge University Press: Cambridge, UK, 1987.

9. Ambjorn, J.; Goerlich, A.; Jurkiewicz, J.; Loll, R. Nonperturbative quantum gravity. Phys. Rep. 2012, 519, 127. [CrossRef]

10. Ashtekar, A. Non-Perturbative Canonical Gravity; IUCAA Lecture Notes; World Scientific: Syracuse, NY, USA, 1990.

11. Nagy, S. Lectures on renormalization and asymptotic safety. Ann. Phys. 2014, 310-346. [CrossRef]

12. Ashtekar, A.; Singh, P. Loop quantum cosmology: A status report. Class. Quant. Grav. 2011, 28, 213001. [CrossRef]

13. Halliwell, J.J. Quantum Cosmology and Baby Universes; Coleman, S., Hartle, J.B., Piran, T., Weinberg, S., Eds.; World Scientific: Singapore, 1991.

14. East, W.E.; Kleban, M.; Linde, A.; Senatore, L. Beginning inflation in an inhomogeneous universe. J. Cosmol. Astropart. Phys. 2016, 9, 010. [CrossRef]

15. Ijjas, A.; Cook, W.G.; Pretorius, F.; Steinhardt, P.J.; Davies, E.Y. Robustness of slow contraction to cosmic initial conditions. J. Cosmol. Astropart. Phys. 2020, 8, 030. [CrossRef]

16. Carter, B. Anthropic principle in cosmology. arXiv 2006, arXiv:gr-qc/0606117.

17. Vilenkin, A.; Yamada, M. Tunneling wave function of the universe. Phys. Rev. D 2018, 98, 066003. [CrossRef]

18. Halliwell, J.J.; Hartle, J.B.; Hertog, T. What is the no-boundary wave function of the Universe? Phys. Rev. D 2019, 99, 043526. [CrossRef]

19. Starobinsky, A.A. Relict gravitation radiation spectrum and initial state of the universe. JETP Lett. 1979 30,682.

20. Guth, A. Inflationary universe: A possible solution to the horizon and flatness problems. Phys. Rev. D 1981, 23, 347. [CrossRef] 
21. Linde, A. A new inflationary universe scenario: A possible solution of the horizon, flatness, homogeneity, isotropy and primordial monopole problems. Phys. Lett. B 1982, 108, 389. [CrossRef]

22. Mukhanov, V.; Chibisov, G. Quantum fluctuations and a nonsingular universe. JETP Lett. 1981, 33, 532.

23. Planck Collaboration; Aghanim, N.; Akrami, Y.; Ashdown, M.; Aumont, J.; Baccigalupi, C.; Ballardini, M.; Banday, A.J.; Barreiro, R.B.; Bartolo, N.; et al. Planck 2018 results. VI. Cosmological parameters. Astron. Astrophys. 2020, 641 , A6.

24. Murphy, G. Big-bang model without singularities. Phys. Rev. D 1973, 8, 4231. [CrossRef]

25. Novello, M.; Salim, J.M. Nonlinear photons in the universe. Phys. Rev. D 1979, 20, 377. [CrossRef]

26. Melnikov, V.; Orlov, S. Nonsingular cosmology as a quantum vacuum effect. Phys. Lett. A 1979, 70, 263. [CrossRef]

27. Khoury, J.; Ovrut, B.A.; Steinhardt, P.J.; Turok, N. Ekpyrotic universe: Colliding branes and the origin of the hot big bang. Phys. Rev. D 2001, 64, 123522. [CrossRef]

28. Khoury, J.; Steinhardt, P.J.; Turok, N. Designing cyclic universe models. Phys. Rev. Lett. 2004, 92, 031302. [CrossRef]

29. Biswas, T.; Brandenberger, R.; Mazumdar, A.; Siegel, W. Non-perturbative gravity, the Hagedorn bounce and the cosmic microwave background. J. Cosmol. Astropart. Phys. 2007, 712, 011. [CrossRef]

30. Brandenberger, R.; Zhang, X. The matter bounce curvaton scenario. J. Cosmol. Astropart. Phys. 2011, 1103, 003.

31. Fabris, J.C.; Perez, R.S.; Bergliaffa, S.E.P.; Pinto-Neto, N. Born-Infeld-like f(R) gravity. Phys. Rev. D 2012, 86, 103525. [CrossRef]

32. Cai, Y.; Easson, D.A.; Brandenberger, R. Towards a nonsingular bouncing cosmology. J. Cosmol. Astropart. Phys. 2012, 8, 020. [CrossRef]

33. Ilyas, A.; Zhu, M.; Zheng, Y.; Cai, Y.; Saridakis, E.N. DHOST bounce. J. Cosmol. Astropart. Phys. 2020, 9, 002. [CrossRef]

34. Bojowald, M. Absence of a singularity in loop quantum cosmology. Phys. Rev. Lett. 2001, 86, 5227. [CrossRef] [PubMed]

35. Ashtekar, A.; Pawlowski, T.; Singh, P. Quantum nature of the big bang. Phys. Rev. Lett. 2006, 14, 141301. [CrossRef] [PubMed]

36. Gielen, S.; Turok, N. Perfect quantum cosmological bounce. Phys. Rev. Lett. 2016, 117, 021301. [CrossRef]

37. Gryb, S.; Thebault, K.P.Y. Superpositions of the cosmological constant allow for singularity resolution and unitary evolution in quantum cosmology. Phys. Lett. 2018, 784, 324. [CrossRef]

38. de Barros, J.A.; Pinto-Neto, N.; Sagioro-Leal, M.A. The causal interpretation of dust and radiation fluid non-singular quantum cosmologies. Phys. Lett. A 1998, 241, 229. [CrossRef]

39. Alvarenga, F.G.; Fabris, J.C.; Lemos, N.A.; Monerat, G.A. Quantum cosmological perfect fluid models. Gen. Relativ. Gravit. 2002, 34, 651. [CrossRef]

40. Novello, M.; Bergliaffa, S.E.P. Bouncing cosmologies. Phys. Rep. 2008, 463, 127. [CrossRef]

41. Brandenberger, R.; Peter, P. Bouncing Cosmologies: Progress and Problems. arXiv 2016, arXiv:1603.05834.

42. Tolman, R.C. On the theoretical requirements for a periodic behaviour of the universe. Phys. Rev. 1931, 38, 1758. [CrossRef]

43. Bohr, N. Can quantum-mechanical description of physical reality be considered complete? Phys. Rev. 1935, 48, 696. [CrossRef]

44. Heisenberg, W. The Physical Principles of the Quantum Theory; Dover: New York, NY, USA, 1949.

45. von Neumann, J. Mathematical Foundations of Quantum Mechanics; Princeton University Press: Princeton, NJ, USA, 1955.

46. Ghirardi, G.C.; Rimini, A.; Weber, T. Unified dynamics for microscopic and macroscopic systems. Phys. Rev. D 1986, 34, 470. [CrossRef]

47. Penrose, R. Quantum Implications: Essays in Honour of David Bohm; Hiley, B.J., Peat, F.D., Eds.; Routledge: London, UK, 1987.

48. DeWitt, B.S.; Graham, N. (Eds.) The Many-Worlds Interpretation of Quantum Mechanics; Princeton University Press: Princeton, USA, 1973.

49. Bohm, D. A suggested interpretation of the quantum theory in terms of "hidden" variables. I. Phys. Rev. 1952, 85, 166. [CrossRef]

50. Holland, P.R. The Quantum Theory of Motion: An Account of the de Broglie-Bohm Causal Interpretation of Quantum Mechanichs; Cambridge University Press: Cambridge, UK, 1993.

51. Dürr, D.; Teufel, S. Bohmian Mechanics; Springer: Berlin, Germany, 2009.

52. Struyve, W. Pilot-wave approaches to quantum field theory. J. Phys. Conf. Ser. 2011, 306, 012047. [CrossRef]

53. Vink, J.C. Quantum potential interpretation of the wave function of the universe. Nucl. Phys. B 1992, 369, 707-728. [CrossRef]

54. Shtanov, Y.V. Pilot wave quantum cosmology. Phys. Rev. D 1996, 54, 2564-2570. [CrossRef] [PubMed]

55. Pinto-Neto, N.; Santini, E.S. Must quantum spacetimes be Euclidean? Phys. Rev. D 1999, 59, 123517. [CrossRef]

56. DeWitt, B.S. Quantum theory of gravity. II. The manifestly covariant theory. Phys. Rev. 1967, 160, 1113. [CrossRef]

57. Valentini, A. Signal-locality, uncertainty, and the subquantum H-theorem. I. Phys. Lett. A 1991, 156, 5-11. [CrossRef]

58. Towler, M.D.; Russell, N.J.; Valentini, A. Time scales for dynamical relaxation to the Born rule. Proc. R. Soc. A 2012, 468, 990-1013. [CrossRef]

59. Norsen, T.; Struyve, W. Weak measurement and Bohmian conditional wave functions. Ann. Phys. 2014, 350, 166-178. [CrossRef]

60. Frolov, V.P.; Vilkovisky, G.A. Spherically symmetric collapse in quantum gravity. Phys. Lett. B 1981, 106, 307. [CrossRef]

61. Casadio, R. Quantum gravitational fluctuations and the semiclassical limit. Int. J. Mod. Phys. D 2000, 9, 51. [CrossRef]

62. Lyth, D.H.; Liddle, A.R. The Primordial Density Perturbation; Cambridge University Press: Cambridge, UK, 2009.

63. Mukhanov, V. Physical Foundations of Cosmology; Cambridge University Press: Cambridge, UK, 2005.

64. Weinberg, S. Cosmology; Oxford University Press: Oxford, UK, 2008.

65. De Unánue, A.; Sudarsky, D. Phenomenological analysis of quantum collapse as source of the seeds of cosmic structure. Phys. Rev. D 2008, 78, 043510. [CrossRef] 
66. Pinto-Neto, N.; Santos, G.; Struyve, W. Quantum-to-classical transition of primordial cosmological perturbations in de BroglieBohm quantum theory. Phys. Rev. D 2012, 85, 083506. [CrossRef]

67. Pinto-Neto, N.; Santos, G.; Struyve, W. Quantum-to-classical transition of primordial cosmological perturbations in de BroglieBohm quantum theory: The bouncing scenario. Phys. Rev. D 2014, 89, 023517. [CrossRef]

68. Hawking, S.W.; Ellis, G.F.R. The Large Scale Structure of Space-Time; Cambridge University Press: Cambridge, UK, 1973.

69. Colistete, R., Jr.; Fabris, J.C.; Pinto-Neto, N. Gaussian superpositions in scalar-tensor quantum cosmological models. Phys. Rev. D 2000, 62, 083507. [CrossRef]

70. Pinto-Neto, N.; Santini, E.S.; Falciano, F.T. Quantization of Friedmann cosmological models with two fluids: Dust plus radiation. Phys. Lett. A 2005, 344, 131-143. [CrossRef]

71. Peter, P.; Pinto-Neto, N. Cosmology without inflation. Phys. Rev. D 2008, 78, 063506. [CrossRef]

72. Halliwell, J.J.; Hawking, S. Origin of structure in the universe. Phys. Rev. D 1985, 31, 1777-1791. [CrossRef]

73. Peter, P.; Pinho, E.J.C.; Pinto-Neto, N. Tensor perturbations in quantum cosmological backgrounds. J. Cosmol. Astropart. Phys. 2005, 7, 014. [CrossRef]

74. Peter, P.; Pinho, E.J.C.; Pinto-Neto, N. Scalar and vector perturbations in quantum cosmological backgrounds. Phys. Rev. D 2007, 76, 023506 .

75. Peter, P.; Pinho, E.J.C.; Pinto-Neto, N. Gravitational wave background in perfect fluid quantum cosmologies. Phys. Rev. D 2006, 73, 104017. [CrossRef]

76. Mukhanov, V.; Feldman, H.A.; Brandenberger, R.H. Theory of cosmological perturbations. Phys. Rep. 1992, 215, 203. [CrossRef]

77. Allen, L.E.; Wands, D. Cosmological perturbations through a simple bounce. Phys. Rev. D 2004, 70, 063515. [CrossRef]

78. Peter, P.; Pinto-Neto, N.; Vitenti, S.D.P. Quantum cosmological perturbations of multiple fluids. Phys. Rev. D 2016, 93, 023520. [CrossRef]

79. Vitenti, S.D.P.; Pinto-Neto, N. Large adiabatic scalar perturbations in a regular bouncing universe. Phys. Rev. D 2012, 85, 023524. [CrossRef]

80. Falciano, F.T.; Pinto-Neto, N.; Vitenti, S.D.P. Scalar field perturbations with arbitrary potentials in quantum backgrounds. Phys. Rev. D 2013, 87, 103514. [CrossRef]

81. Falciano, F.T.; Pinto-Neto, N. Scalar perturbations in scalar field quantum cosmology. Phys. Rev. D 2009, 79, 023507. [CrossRef]

82. Bessada, D.; Pinto-Neto, N.; Siffert, B.B.; Miranda, O.D. Stochastic background of relic gravitons in a bouncing quantum cosmological model. J. Cosmol. Astropart. Phys. 2012, 54, 1211. [CrossRef]

83. Quintin, J.; Sherkatghanad, Z.; Cai, Y.-F.; Brandenberger, R.H. Evolution of cosmological perturbations and the production of non-Gaussianities through a nonsingular bounce: Indications for a no-go theorem in single field matter bounce cosmologies. Phys. Rev. D 2015, 92, 063532. [CrossRef]

84. Li, Y.-B.; Quintin, J.; Wang, D.-G.; Cai, Y.-F. Matter bounce cosmology with a generalized single field: Non-Gaussianity and an extended no-go theorem. J. Cosmol. Astropart. Phys. 2017, 3, 031. [CrossRef]

85. Finelli, F.; Brandenberger, R. Generation of a scale-invariant spectrum of adiabatic fluctuations in cosmological models with a contracting phase. Phys. Rev. D 2002, 65, 103522. [CrossRef]

86. Pinto-Neto, N.; Vitenti, S.D.P. Comment on "Growth of covariant perturbations in the contracting phase of a bouncing universe". Phys. Rev. D 2014, 89, 028301. [CrossRef]

87. Pinto-Neto, N.; Fabris, J.C.; Toniato, J.D.; Vicente, G.S.; Vitenti, S.D.P. Vector perturbations in bouncing cosmology. Phys. Rev. D 2020, 101, 123519. [CrossRef]

88. Maier, R.; Pereira, S.; Pinto-Neto, N.; Siffert, B.B. Bouncing models with a cosmological constant. Phys. Rev. D 2012, 85, 023508. [CrossRef]

89. Bacalhau, A.P.; Pinto-Neto, N.; Vitenti, S.D.P. Consistent scalar and tensor perturbation power spectra in single fluid matter bounce with dark energy era. Phys. Rev. D 2018, 97, 083517. [CrossRef]

90. Heard, I.P.C.; Wands, D. Cosmology with positive and negative exponential potentials. Class. Quantum Gravity 2002, $19,5435$. [CrossRef]

91. Colin, S.; Pinto-Neto, N. Quantum matter bounce with a dark energy expanding phase. Phys. Rev. D 2017, 96, 063502. [CrossRef]

92. Lehners, J.; Steinhardt, P.J. Dark energy and the return of the phoenix universe. Phys. Rev. D 2009, 79, 063503. [CrossRef]

93. Cai, Y.; Marciano, A.; Wang, D.; Wilson-Ewing, E. Bouncing cosmologies with dark matter and dark energy. Universe 2017, 3, 1. [CrossRef]

94. Odintsov, S.D.; Oikonomou, V.K.; Paul, T. From a Bounce to the Dark Energy Era with F(R) Gravity arXiv 2020, arXiv:2009.09947.

95. Celani, D.; Pinto-Neto, N.; Vitenti, S.D.P. Particle creation in bouncing cosmologies. Phys. Rev. D 2017, 95, 023523. [CrossRef]

96. Scardua, A.; Guimarães, L.F.; Pinto-Neto, N.; Vicente, G.S. Fermion production in bouncing cosmologies. Phys. Rev. D 2018, 98 , 083505. [CrossRef]

97. Olive, K.A. Review of particle physics. Chin. Phys. C 2014, 38, 090001. [CrossRef]

98. Cohen, A.G.; Rujula, A.D.; Glashow, S.L. A matter-antimatter universe? Astrophys. J. 1998, 495, 539. [CrossRef]

99. Sakharov, A.D. Violation of CP Invariance, Casymmetry, and baryon asymmetry of the universe. JETP Lett. 1967, 5, 24.

100. Kuzmin, V.A.; Rubakov, V.A.; Shaposhnikov, M.E. On anomalous electroweak baryon-number non-conservation in the early universe. Phys. Lett. 1985, 155B, 36. [CrossRef] 
101. Shaposhnikov, M.E. Possible appearance of the baryon asymmetry of the universe in an electroweak theory. JETP Lett. 1986, $44,465$.

102. Shaposhnikov, M.E. Baryon asymmetry of the universe in standard electroweak theory. Nucl. Phys. 1987, 287, 757. [CrossRef]

103. Antunes, V.; Bediaga, I.; Novello, M. Gravitational baryogenesis without CPT violation. J. Cosmol. Astropart. Phys. 2019, 10, 076. [CrossRef]

104. Cohen, A.G.; Kaplan, D.B. Thermodynamic generation of the baryon asymmetry. Phys. Lett. B 1987, 199, 251-258. [CrossRef]

105. Simone, A.D.; Kobayashi, T. Cosmological aspects of spontaneous baryogenesis. J. Cosmol. Astropart. Phys. 2016, 8, 052. [CrossRef]

106. Davoudiasl, H.; Kitano, R.; Kribs, G.D.; Murayama, H.; Steinhardt, P.J. Gravitational baryogenesis. Phys. Rev. Lett. 2004, 93, 201301. [CrossRef] [PubMed]

107. Odintsov, S.D.; Oikonomou, V.K. Loop quantum cosmology gravitational baryogenesis. EPL Europhys. Lett. $2016,116,49001$. [CrossRef]

108. Delgado, P.C.M.; Jesus, M.B.; Pinto-Neto, N.; Mourão, T.; Vicente, G.S. Baryogenesis in cosmological models with symmetric and asymmetric quantum bounces. Phys. Rev. D 2020, 102, 063529. [CrossRef]

109. Brandenburg, A.; Subramanian, K. Astrophysical magnetic fields and nonlinear dynamo theory. Phys. Rept. 2005, 417, 1. [CrossRef]

110. Durrer, R.; Neronov, A. Cosmological magnetic fields: Their generation, evolution and observation. Astron. Astrophys. Rev. 2013, 21, 62. [CrossRef]

111. Pogosian, L.; Zucca, A. Searching for primordial magnetic fields with CMB B-modes. Class. Quant. Grav. 2018, $35,124004$. [CrossRef]

112. Turner, M.S.; Widrow, L.M. Inflation-produced, large-scale magnetic fields. Phys. Rev. D 1988, 37, 2743. [CrossRef]

113. Campanelli, L.; Cea, P.; Fogli, G.L.; Tedesco, L. Inflation-produced magnetic fields in $R^{n} F^{2}$ and $I F^{2}$ models. Phys. Rev. D 2008, 77, 123002. [CrossRef]

114. Kunze, K.E. Completing magnetic field generation from gravitationally coupled electrodynamics with the curvaton mechanism. Phys. Rev. D 2013, 87, 063505. [CrossRef]

115. Subramanian, K. The origin, evolution and signatures of primordial magnetic fields. Rept. Prog. Phys. 2016, 79, 076901. [CrossRef]

116. Demozzi, V.; Mukhanov, V.; Rubinstein, H. Magnetic fields from inflation? J. Cosmol. Astropart. Phys. 2009, 908, 025. [CrossRef]

117. Green, D.; Kobayashi, T. Constraints on primordial magnetic fields from inflation. J. Cosmol. Astropart. Phys. $2016,3,010$. [CrossRef]

118. Drummond, I.; Hathrell, S. QED vacuum polarization in a background gravitational field and its effect on the velocity of photons. Phys. Rev. D 1980, 22, 343. [CrossRef]

119. Frion, E.; Pinto-Neto, N.; Vitenti, S.D.P.; Bergliaffa, S.E.P. Primordial magnetogenesis in a bouncing universe. Phys. Rev. D 2020, 101, 103503. [CrossRef]

120. Qian, P.; Cai, Y.-F.; Easson, D.A.; Guo, Z.-K. Magnetogenesis in bouncing cosmology. Phys. Rev. D 2016, 94, 083524. [CrossRef]

121. Membiela, F.A. Primordial magnetic fields from a non-singular bouncing cosmology. Nucl. Phys. B 2014, 885, 196. [CrossRef]

122. Sriramkumar, L.; Atmjeet, K.; Jain, R.K. Generation of scale invariant magnetic fields in bouncing universes. J. Cosmol. Astropart. Phys. 2015, 9, 010. [CrossRef]

123. Durrer, R.; Caprini, C. Primordial magnetic fields and causality. J. Cosmol. Astropart. Phys. 2003, 11, 010. [CrossRef]

124. Taylor, A.; Vovk, I.; Neronov, A. Extragalactic magnetic fields constraints from simultaneous GeV-TeV observations of blazars. Astron. Astrophys. A 2011, 144, 529. [CrossRef]

125. Minoda, T.; Tashiro, H.; Takahashi, T. Insight into primordial magnetic fields from 21-cm line observation with EDGES experiment. Mon. Not. R. Astron. Soc. 2019, 488, 2001. [CrossRef]

126. Belinskii, V.; Lifshitz, E.; Khalatnikov, I.M. Oscillatory approach to the singular point in relativistic cosmology. Usp. Fiz. Nauk 1970, 102, 463. [CrossRef]

127. Bergeron, H.; Dapor, A.; Gazeau, J.P.; Malkiewicz, P. Smooth big bounce from affine quantization. Phys. Rev. D 2014, 89, 083522. [CrossRef]

128. Cai, Y.-F.; Xue, W.; Brandenberger, R.; Zhang, X. Non-Gaussianity in a matter bounce. J. Cosmol. Astropart. Phys. $2009,5,011$. [CrossRef]

129. Agullo, I.; Bolliet, B.; Sreenath, V. Non-Gaussianity in loop quantum cosmology. Phys. Rev. D 2018, 97, 066021. [CrossRef] 